\title{
Search for Higgs portal DM at the ILC
}

\author{
P. Ko ${ }^{a, b}$ and Hiroshi Yokoya ${ }^{b}$ \\ ${ }^{a}$ School of Physics, KIAS, \\ Seoul 02455, Korea \\ ${ }^{b}$ Quantum Universe Center, KIAS, \\ Seoul 02455, Korea \\ E-mail: pko@kias.re.kr, hyokoya@kias.re.kr
}

ABSTRACT: Higgs portal dark matter (DM) models are simple interesting and viable DM models. There are three types of the models depending on the DM spin: scalar, fermion and vector DM models. In this paper, we consider renormalizable, unitary and gauge invariant Higgs portal DM models, and study how large parameter regions can be surveyed at the International Linear Collider (ILC) experiment at $\sqrt{s}=500 \mathrm{GeV}$. For the Higgs portal singlet fermion and vector DM cases, the force mediator involves two scalar propagators, the SM-like Higgs boson and the dark Higgs boson. We show that their interference generates interesting and important patterns in the mono- $Z$ plus missing $E_{T}$ signatures at the ILC, and the results are completely different from those obtained from the Higgs portal DM models within the effective field theories. In addition, we show that it would be possible to distinguish the spin of DM in the Higgs portal scenarios, if the shape of the recoil-mass distribution is observed. We emphasize that the interplay between these collider observations and those in the direct detection experiments has to be performed in the model with renomalizability and unitarity to combine the model analyses in different scales.

Keywords: Beyond Standard Model, Dark matter, e+-e- Experiments, Higgs physics

ARXIV EPRINT: 1603.04737 


\section{Contents}

1 Introduction 1

2 Model Lagrangians $\quad 3$

2.1 Singlet scalar DM with Higgs portal 4

2.2 Model for singlet fermion DM with Higgs portal 5

2.3 Gauge invariant unitary model for vector DM with Higgs portal 7

2.4 Comparison of three models 8

3 How to distinguish 3 cases at the ILC? 9

4 Parameter constraints $\quad 11$

$4.1 m_{D} \leq m_{H_{1}} / 2 \quad 11$

$4.2 m_{D} \geq m_{H_{1}} / 2$

4.2.1 Scalar DM, $m_{D} \geq m_{H_{2}} / 2$ or $m_{H_{2}} \geq \sqrt{s}-m_{Z}$ cases $\quad 14$

4.2.2 Fermion and vector DM models with $m_{D} \leq m_{H_{2}} / 2 \quad 17$

$\begin{array}{lll}4.3 & \text { Comparison with Higgs portal DM models within EFT } & 19\end{array}$

5 Conclusion $\quad 20$

\section{Introduction}

One of the most pressing question after the Higgs boson discovery is to understand particle physics nature of nonbaryonic dark matter (DM) of the universe. Existence of DM has been confirmed only through gravitation probes, and it is important to find their properties (mass, spin, quantum numbers, etc.) using terrestrial experimental apparatus. Among many DM models, Higgs portal scalar, fermion and vector DM models are simple and interesting [1-8], which are also phenomenologically viable and have intimate connections to the observed $125 \mathrm{GeV}$ Higgs boson [9-11]. Study on the characteristic signatures at collider experiments has to be performed to verify these models. In particular it would be important to know if one can distinguish the DM spin at current or future colliders.

In this work, we present a detailed study on this issue at the International Linear Collider (ILC) [12-14] in a careful manner using the Higgs portal DM models that are renormalizable, gauge invariant and unitary [15-18]. For high-energy collider studies, using an effective field theory (EFT) could be dangerous, especially when we do not know the mass scales of new degrees of freedom. This is especially true for the dark matter physics, since nothing is known about the DM mass, their interactions among themselves and with the SM particles, as well as the masses of dark force mediators such as a dark photon or the dark Higgs boson. 
Earlier analyses of this issue are based on the following model Lagrangians [3, 19-21]:

$$
\begin{aligned}
\mathcal{L}_{\mathrm{SSDM}}^{\mathrm{EFT}} & =\frac{1}{2} \partial_{\mu} S \partial^{\mu} S-\frac{1}{2} m_{S}^{2} S^{2}-\frac{\lambda_{S}}{4 !} S^{4}-\frac{\lambda_{H S}}{2} S^{2} H^{\dagger} H \\
\mathcal{L}_{\mathrm{SFDM}}^{\mathrm{EFT}} & =\bar{\chi}\left(i \not \partial-m_{\chi}\right) \chi-\frac{\lambda_{\chi} H}{\Lambda} \bar{\chi} \chi H^{\dagger} H \\
\mathcal{L}_{\mathrm{VDM}}^{\mathrm{EFT}} & =-\frac{1}{4} V_{\mu \nu} V^{\mu \nu}+\frac{1}{2} m_{V}^{2} V_{\mu} V^{\mu}-\frac{\lambda_{V H}}{2} V_{\mu} V^{\mu} H^{\dagger} H-\frac{\lambda_{V}}{4}\left(V_{\mu} V^{\mu}\right)^{2}
\end{aligned}
$$

The Lagrangian for scalar DM (1.1) is renormalizable and unitary, and one can study scalar DM phenomenology without any limitation. ${ }^{1}$ On the other hand, the Lagrangians for singlet fermion DM (1.2) contains a dim-5 operator Higgs portal interaction, and eventually one has to consider its UV completions. The simplest UV completion for the singlet fermion DM model with Higgs portal have been constructed in refs. $[15,16]$ by introducing a real singlet scalar mediator that couples to singlet fermion DM and also to the SM Higgs fields in an $\mathrm{SU}(2)$ singlet combination, $H^{\dagger} H$. Both DM phenomenology and vacuum structures of the model have been studied in great detail. After electroweak symmetry breaking, the singlet scalar mixes with the SM Higgs boson, which plays an important role in DM direct detections as well as DM searches at colliders.

Likewise, the Higgs portal vector DM (VDM) model is problematic, because it does not respect either unitarity or gauge invariance since the VDM mass is given by hand. Such drawbacks could be overcome in UV-complete VDM models [17, 18, 24, 25], where VDM becomes a dark gauge boson associated with a local $\mathrm{U}(1)_{X}$ dark gauge symmetry. VDM gets massive through a dark Higgs mechanism, and there would be a mixing between the SM Higgs boson and the dark Higgs boson. Then VDM becomes stable by ad hoc $Z_{2}$ symmetry plus charge conjugation symmetry under $\mathrm{U}(1)_{X}$. One can also construct models where VDM becomes absolutely stable due to the unbroken dark gauge symmetry, which has much richer structure in the dark sector, namely dark monopole, massless dark photon and dark Higgs boson as well as VDM [26].

These models have a new degree of freedom, a singlet-like scalar boson, which mixes with the SM Higgs boson. Therefore at least two more parameters appear: the mass of the 2nd scalar boson $m_{H_{2}}$ and the mixing angle $\alpha$, as well as the coupling between the singlet scalar and DM. One of the authors utilizes the 2nd scalar in order to explain the galactic center $\gamma$-ray excess $[22,27-29]$ and to obtain a larger tensor-to-scalar ratio in the Higgs portal assisted Higgs inflation scenario [30]. Also it was shown that the correlation between the upper bound on the invisible Higgs decay branching ratio and the upper bound on the direct detection cross section is modified in the renormalizable, unitary and gauge invariant models [31]. Global analysis of the SM Higgs signal strengths should include its possible mixing with a singlet scalar in this kind of DM models [32-34]

The shortcomings of these effective Lagrangians for singlet fermion and vector DM cases have been pointed out within the DM phenomenology $[15,16]$. Especially the directdetection cross-section (DM-nucleon scattering cross-section) depends on the model La-

\footnotetext{
${ }^{1}$ There is an issue about Planck-scale suppressed $Z_{2}$ breaking operator which would make EW scale scalar DM decay fast. We refer to ref. [22] for implementing global $Z_{2}$ to $Z_{2}$ subgroup of U(1) dark gauge symmetry which resolves this problem. See also ref. [23] for comparison of global vs. local $Z_{3}$ scalar DM models.
} 
grangians, namely there is a generic destructive interference between the SM Higgs boson and the second singlet-like scalar boson.

One important question in the Higgs portal DM models is how to distinguish three different cases at colliders and/or other experiments. In this paper, we study this issue at the ILC, postponing the same issue at the LHC for future publication [35]. At the ILC, we can fix the initial beam energy and measure the 4-momenta of the final $Z^{0}$ in the process $e^{+} e^{-} \rightarrow Z^{0}+\mathbb{E}_{T}$, and there are simple relations among $E_{Z}, M_{D D}$ and $\mathbb{E}_{T}$ :

$$
\begin{aligned}
M_{D D}^{2} & =s+m_{Z}^{2}-2 E_{Z} \sqrt{s}, \\
\mathbb{E}_{T} & =\frac{s-m_{Z}^{2}+M_{D D}^{2}}{2 \sqrt{s}}, \\
E_{Z} & =\frac{s+m_{Z}^{2}-M_{D D}^{2}}{2 \sqrt{s}},
\end{aligned}
$$

where $\sqrt{s}$ is the total collision energy in the laboratory frame, $M_{D D}$ is the invariant mass squared of the DM pair ( $D=S, \chi, V$ in the following sections). Therefore one can reconstruct all the relevant kinematic variables related with DM, $M_{D D}^{2}$ and $\mathbb{E}_{T}$ at the ILC, which renders us to study the Higgs portal DM properties in clean ways.

This paper is organized as follows. In section 2, we describe the Higgs portal DM models for scalar, fermion and vector DM. We set up the renormalizable, unitary and gauge invariant Lagrangians, which can be used at an arbitrarily high-energy scale relevant to colliders, and often produce different results from the Higgs portal DM models with effective nonrenormalizable and gauge-variant interactions. First, we list the processes for the DM production at the ILC in the Higgs portal DM models. Then, we present the detail analysis for the relevant process:

$$
e^{+}\left(p_{1}\right)+e^{-}\left(p_{2}\right) \rightarrow h^{*}(q)+Z\left(p_{Z}\right) \rightarrow S\left(k_{1}\right)+S\left(k_{2}\right)+Z\left(p_{Z}\right),
$$

for the scalar DM case, and the counter processes for the fermion and vector DM models. In particular there are two scalar propagators contributing to this process for the fermion and vector DM cases, as first pointed out in ref. [36]. Then in section 3, we give qualitative discussions on how to distinguish 3 different cases with the rate and shape analysis. In section 4, we describe the detailed analysis on the parameter constraints at the ILC at $\sqrt{s}=500 \mathrm{GeV}$, and compare our results with those obtained from the Higgs portal DM models based on the effective field theories. Finally we conclude our analysis in section 5 .

\section{Model Lagrangians}

In this section, we define the simplified models for Higgs portal DM, where DM can be either a scalar, fermion or vector particle. It is important to start from model Lagrangians that are unitary and renormalizable and invariant under full SM gauge symmetry.

At the ILC, Higgs portal DM can be produced through the following processes [37-41]:

$$
e^{+} e^{-} \rightarrow\left\{\begin{array}{cc}
Z^{0} H_{i=1,2}, & \text { (Higgs-strahlung }), \\
\nu_{e} \overline{\nu_{e}} H_{i=1,2}, & \left(W^{+} W^{-} \text {fusion }\right), \\
e^{+} e^{-} H_{i=1,2}, & \left(Z^{0} Z^{0} \text { fusion }\right),
\end{array}\right.
$$


all of which are followed by the $H_{i=1,2} D D$ couplings. Note that as we will see in the following, $H_{1}$ and $H_{2}$ are the Higgs bosons portal to DM in the fermion and vector DM models. There is no $H_{2}$ and $H_{1}=H_{\mathrm{SM}}$ in the scalar DM case. One can identify the 1st process by tagging $Z^{0}$ in the $\mu^{+} \mu^{-}$or $q \bar{q}$ channels. The 2 nd process is impossible to observe since there is no detectable particle in the final-state. The 3rd process has a unique event topology too, but the total cross-section is more than 10 times smaller than the 1st process up to $\sqrt{s} \simeq 3 \mathrm{TeV}[38]$. Therefore, the 1 st process is the most promising process to observe at the ILC with the current proposal [37, 39].

In the following, we consider the 1st process as the signal of the DM production in the Higgs portal DM models. However, depending on the details of the models which satisfy the gauge invariance, unitarity and renormalizability, as well as on the model parameter regions, the collider signatures can be different. Thus, we have to perform the careful study on these detectability.

\subsection{Singlet scalar DM with Higgs portal}

In the scalar DM case, the model is very simple:

$$
\mathcal{L}_{\mathrm{SSDM}}=\frac{1}{2} \partial_{\mu} S \partial^{\mu} S-\frac{1}{2} m_{0}^{2} S^{2}-\lambda_{H S} H^{\dagger} H S^{2}-\frac{\lambda_{S}}{4 !} S^{4} .
$$

The terms with odd numbers of $S$ is restricted by imposing $Z_{2}$ symmetry under which $S$ changes sign, but all the SM particles do not. Then $S$ will be stable and can make a good DM candidate.

From this Lagrangian, we can calculate the amplitude for the process, (1.4):

$$
\begin{aligned}
i \mathcal{M}_{S}= & \bar{v}\left(p_{2}, \lambda_{2}\right)\left(-i \frac{g_{Z}}{2}\right)\left[c_{V}^{e} \gamma^{\mu}-c_{A}^{e} \gamma^{\mu} \gamma_{5}\right] u\left(p_{1}, \lambda_{1}\right) \cdot \frac{-i\left(g_{\mu \nu}-\frac{P_{\mu} P_{\nu}}{m_{Z}^{2}}\right)}{s-m_{Z}^{2}+i m_{Z} \Gamma_{Z}} \\
& \cdot i g^{\nu \alpha} \frac{2 m_{Z}^{2}}{v} \epsilon_{\alpha}\left(p_{Z}\right) \times\left[\frac{i}{t-m_{h}^{2}+i m_{h} \Gamma_{h}} \cdot 2 i \lambda_{H S} v\right] .
\end{aligned}
$$

We define $P^{\mu}=p_{1}^{\mu}+p_{2}^{\mu}, c_{V}^{e}=-1 / 2+2 s_{W}^{2}$ with $s_{W}=\sin \theta_{W}$ where $\theta_{W}$ is the weak mixing angle, and $c_{A}^{e}=-1 / 2$. Then the amplitude $\mathcal{M}_{S}$ can be factorized into two parts:

$$
\mathcal{M}_{S}=\mathcal{M}_{h^{*} Z} \cdot \frac{2 \lambda_{H S} v}{t-m_{h}^{2}+i m_{h} \Gamma_{h}} .
$$

The squared amplitude for the $h^{*} Z$ production part is

$$
\left|\mathcal{M}_{h^{*} Z}\right|^{2}=\mathcal{P}\left(\lambda_{1}, \lambda_{2}\right) \frac{8 m_{Z}^{4}}{v^{4}}\left|r_{Z}(s)\right|^{2}\left(\frac{p_{1} \cdot p_{Z} p_{2} \cdot p_{Z}}{s^{2}}+\frac{m_{Z}^{2}}{s}\right),
$$

where we define $P_{e e}\left(\lambda_{1}, \lambda_{2}\right)=\left(1-\lambda_{1} \lambda_{2}\right)\left(\left|c_{V}^{e}\right|^{2}+\left|c_{A}^{e}\right|^{2}\right)-2\left(\lambda_{1}-\lambda_{2}\right) \operatorname{Re}\left(c_{V}^{e} c_{A}^{e *}\right)$ and $r_{Z}(s)=$ $1 /\left(1-m_{Z}^{2} / s+i m_{Z} \Gamma_{Z} / s\right)$. For the spin-averaged cross-section, $\mathcal{P}_{e e} \rightarrow \overline{\mathcal{P}}_{e e}=\left(\left|c_{V}^{e}\right|^{2}+\left|c_{A}^{e}\right|^{2}\right)$ is adopted. The 3 -body phase-space is given by

$$
\begin{aligned}
d \Phi_{3}\left(p_{1}+p_{2} ; k_{1}, k_{2}, p_{Z}\right) & =\frac{d t}{2 \pi} \cdot d \Phi_{2}\left(p_{1}+p_{2} ; q, p_{Z}\right) \cdot d \Phi_{2}\left(q ; k_{1}, k_{2}\right) \\
& =\frac{d t}{2 \pi} \cdot \frac{\hat{\beta}}{8 \pi} \frac{d \hat{\Omega}}{4 \pi} \cdot \frac{\beta_{S}}{8 \pi} \frac{d \Omega_{S}}{4 \pi},
\end{aligned}
$$


where $t=q^{2}, \hat{\beta}=\lambda^{1 / 2}\left(1, m_{Z}^{2} / s, t / s\right)$ and $\beta_{D}=\lambda^{1 / 2}\left(1, m_{D}^{2} / t, m_{D}^{2} / t\right)=\sqrt{1-4 m_{D}^{2} / t}$ (for $D=S, \chi, V)$, with $\lambda(a, b, c)=a^{2}+b^{2}+c^{2}-2(a b+b c+c a)$. The range of the kinematic variable $t$ is

$$
4 m_{D}^{2} \leq t \leq\left(\sqrt{s}-m_{Z}\right)^{2}
$$

for a given $\sqrt{s}$ (the CM energy of the ILC). $d \hat{\Omega}$ and $d \Omega_{S}$ are two-body phase-space volumes for the $h^{*} Z$ and the $S S$ systems, respectively.

Thus, the cross-section is straightforwardly calculated to be

$$
\begin{aligned}
d \sigma_{S} & =C_{S} \frac{1}{2 s}\left|\mathcal{M}_{S}\right|^{2} d \Phi_{3} \\
& =\frac{d t}{2 \pi} \cdot \frac{1}{2 s}\left|\mathcal{M}_{h^{*} Z}\right|^{2} d \Phi_{2}\left(p_{1}+p_{2} ; q, p_{Z}\right) \cdot C_{S} \frac{\beta_{D}}{8 \pi}\left|\frac{2 \lambda_{H S} v}{t-m_{h}^{2}+i m_{h} \Gamma_{h}}\right|^{2} .
\end{aligned}
$$

Here $C_{S}$ is a symmetric factor, $C_{S}=1 / 2$, taking care of the identical $S$ 's in the final states. By defining the total cross section for $e^{+} e^{-} \rightarrow h^{*} Z$ as

$$
\begin{aligned}
\sigma_{h^{*} Z}(s, t) & =\frac{1}{2 s}\left|\mathcal{M}_{h^{*} Z}\right|^{2} d \Phi_{2}\left(p_{1}+p_{2} ; q, p_{Z}\right) \\
& =\mathcal{P}_{e e}\left(\lambda_{1}, \lambda_{2}\right) \frac{1}{6 s} \frac{m_{Z}^{4}}{v^{4}}\left|r_{Z}(s)\right|^{2} \frac{\hat{\beta}}{8 \pi}\left[\hat{\beta}^{2}+\frac{12 m_{Z}^{2}}{s}\right],
\end{aligned}
$$

and a form factor for the scalar DM production as

$$
G_{S}(t)=C_{S} \frac{\beta_{D}}{8 \pi}\left|\frac{2 \lambda_{H S} v}{t-m_{h}^{2}+i m_{h} \Gamma_{h}}\right|^{2},
$$

the $t$-distribution is given as

$$
\frac{d \sigma_{S}}{d t}=\frac{1}{2 \pi} \sigma_{h^{*} Z}(s, t) \cdot G_{S}(t) .
$$

Note that, at lepton colliders, $t$ is observable from the $Z$-boson momentum by $t=\left(p_{1}+\right.$ $\left.p_{2}-p_{Z}\right)^{2}=s+m_{Z}^{2}-2 \sqrt{s} E_{Z}$ where $E_{Z}$ is the $Z$-boson energy in the C.M. frame of $e^{+} e^{-}$. $\sigma_{h^{*} Z}$ depends on $t$ as well through $\hat{\beta}$.

\subsection{Model for singlet fermion DM with Higgs portal}

In the case of the Higgs portal fermion DM model, we assume that DM is a singlet Dirac fermion $\chi$ with some nontrivial dark charge so that it is distinguishable from right-handed neutrinos. Otherwise one can write down the Dirac neutrino Yukawa terms and $\chi$ would decay and cannot be a good cold DM candidate. The simplest UV-completion of the Higgs portal fermion DM model can be constructed by introducing a $\mathrm{SU}(2)$-singlet scalar which has a vacuum expectation value and a Yukawa interaction to DM giving its mass:

$$
\begin{aligned}
\mathcal{L}_{\mathrm{SFDM}}= & \bar{\chi}\left(i \not \partial-m_{\chi}-y_{\chi} \phi\right) \chi+\frac{1}{2} \partial_{\mu} \phi \partial^{\mu} \phi-\frac{1}{2} m_{0}^{2} \phi^{2} \\
& -\lambda_{H \phi} H^{\dagger} H \phi^{2}-\mu_{\phi} \phi H^{\dagger} H-\mu_{0}^{3} \phi-\frac{\mu_{\phi}}{3 !} \phi^{3}-\frac{\lambda_{\phi}}{4 !} \phi^{4}
\end{aligned}
$$


Expanding both fields around their VEVs by $H \rightarrow\left(0,\left(v_{H}+h\right) / \sqrt{2}\right)^{\top}$ and $\phi \rightarrow v_{\phi}+\phi$, we can derive the Lagrangian in terms of $h$ and $\phi$. After diagonalization of the mass matrix in the scalar sector,

$$
\left(\begin{array}{l}
h \\
\phi
\end{array}\right)=\left(\begin{array}{cc}
\cos \alpha & \sin \alpha \\
-\sin \alpha & \cos \alpha
\end{array}\right)\left(\begin{array}{l}
H_{1} \\
H_{2}
\end{array}\right),
$$

DM $\chi$ couples with both $H_{1}$ and $H_{2}$. The interaction Lagrangian of $H_{1}$ and $H_{2}$ with the SM fields and DM $\chi$ is given by

$$
\begin{aligned}
\mathcal{L}_{\text {int }}= & -\left(H_{1} \cos \alpha+H_{2} \sin \alpha\right)\left[\sum_{f} \frac{m_{f}}{v_{H}} \bar{f} f-\frac{2 m_{W}^{2}}{v_{H}} W_{\mu}^{+} W^{-\mu}-\frac{m_{Z}^{2}}{v_{H}} Z_{\mu} Z^{\mu}\right] \\
& +y_{\chi}\left(H_{1} \sin \alpha-H_{2} \cos \alpha\right) \bar{\chi} \chi,
\end{aligned}
$$

following the convention of ref. [15]. We identify the observed $125 \mathrm{GeV}$ scalar boson as $H_{1}$. The mixing between $h$ and $\phi$ leads to a universal suppression factor of the Higgs signal strengths at the LHC, independent of production and decay channels [15]. From the current data on Higgs signal strengths and the upper bound on the Higgs invisible branching ratio, one can derive an upper bound, $\sin \alpha \lesssim 0.53$ [42-44]. This bound is still quite weak and should be improved in the future experiments.

Defining $\lambda_{\chi}=y_{\chi} \sin \alpha \cos \alpha$, the scattering amplitude for the process,

$$
e^{+}\left(p_{1}\right)+e^{-}\left(p_{2}\right) \rightarrow H_{1} / H_{2}(q)+Z\left(p_{Z}\right) \rightarrow \chi\left(k_{1}\right)+\bar{\chi}\left(k_{2}\right)+Z\left(p_{Z}\right),
$$

is written as

$$
\mathcal{M}_{\chi}=\mathcal{M}_{h^{*} Z} \cdot \lambda_{\chi}\left[\frac{1}{t-m_{H_{1}}^{2}+i m_{H_{1}} \Gamma_{H_{1}}}-\frac{1}{t-m_{H_{2}}^{2}+i m_{H_{2}} \Gamma_{H_{2}}}\right] \bar{u}\left(k_{1}\right) v\left(k_{2}\right) .
$$

Thus, the squared matrix elements are

$$
\sum\left|\mathcal{M}_{\chi}\right|^{2}=\left|\mathcal{M}_{h^{*} Z}\right|^{2} \lambda_{\chi}^{2}\left|\frac{1}{t-m_{H_{1}}^{2}+i m_{H_{1}} \Gamma_{H_{1}}}-\frac{1}{t-m_{H_{2}}^{2}+i m_{H_{2}} \Gamma_{H_{2}}}\right|^{2} \sum|\bar{u} v|^{2},
$$

where the spin-sum of the fermion DM wave-functions is evaluated to be

$$
\sum|\bar{u} v|^{2}=2\left(t-4 m_{\chi}^{2}\right)=2 t \beta_{\chi}^{2}
$$

Thus, we obtain

$$
\frac{d \sigma_{\chi}}{d t}=\frac{1}{2 \pi} \sigma_{h^{*} Z}(s, t) \cdot G_{\chi}(t)
$$

where

$$
G_{\chi}(t)=C_{\chi} \frac{\beta_{\chi}^{3}}{8 \pi} \cdot 2 \lambda_{\chi}^{2} t \cdot\left|\frac{1}{t-m_{H_{1}}^{2}+i m_{H_{1}} \Gamma_{H_{1}}}-\frac{1}{t-m_{H_{2}}^{2}+i m_{H_{2}} \Gamma_{H_{2}}}\right|^{2} .
$$

The symmetric factor for this case is $C_{\chi}=1$. 


\subsection{Gauge invariant unitary model for vector DM with Higgs portal}

There are a number of different models for stable or long-lived vector DM $V_{\mu}$ with Higgs portal. The simplest model would be a phenomenological model where a discrete $Z_{2}$ symmetry $\left(V_{\mu} \rightarrow-V_{\mu}\right)$ is imposed by hand $[17,18,24]$. In order to construct a renormalizable and unitary model, it is important to assume a dark gauge symmetry $\mathrm{U}(1)_{X}$ and dark Higgs $\Phi$ whose VEV provides a nonzero mass to vector DM $V_{\mu}$ and produces a dark Higgs $\varphi$ as a remnant of the Higgs mechanism:

$$
\mathcal{L}=-\frac{1}{4} V_{\mu \nu} V^{\mu \nu}+D_{\mu} \Phi^{\dagger} D^{\mu} \Phi-\frac{\lambda_{\Phi}}{4}\left(\Phi^{\dagger} \Phi-\frac{v_{\phi}^{2}}{2}\right)^{2}-\lambda_{H \Phi}\left(H^{\dagger} H-\frac{v^{2}}{2}\right)\left(\Phi^{\dagger} \Phi-\frac{v_{\phi}^{2}}{2}\right)
$$

One can also consider more sophisticated models where the aforementioned ad hoc $Z_{2}$ symmetry is implemented to some local dark gauge symmetries. There are basically two different categories in this class.

- VDM is stable due to unbroken gauge symmetry [26]: one of the present authors constructed a hidden sector monopole model where the renowned 't Hooft-Polyakov monopole is put in the hidden sector, which is connected to the SM sector through the Higgs portal interaction. There are two stable DM in this case: hidden monopole which is stable due to topological reason, and vector DM which is stable due to unbroken $\mathrm{U}(1)_{X}$ subgroup. There is massless dark photon associated with unbroken $\mathrm{U}(1)_{X}$, and it can generate strong self-interaction between dark matters, and would contribute to the dark radiation at the level of $\sim 0.1$ which is perfectly consistent with Planck data.

- VDM is stable at renormalizable level, but could decay and is long lived when we consider higher dimensional operators [45]. This happens if the dark gauge group $\mathrm{SU}(2)_{X}$ is completely broken by $\mathrm{SU}(2)_{X}$ doublet complex dark Higgs, for example. In this case the dark gauge symmetry is completely broken, and the massive VDM is not stable due to the presence of higher dimensional nonrenormalizable operators.

In this paper, we do not consider these sophisticated models, and will consider phenomenological VDM models, which could be considered as a simplified VDM model:

$\mathcal{L}_{\mathrm{VDM}}=-\frac{1}{4} V_{\mu \nu} V^{\mu \nu}+\frac{1}{2} m_{V}^{2} V_{\mu} V^{\mu}\left(1+\frac{\varphi}{v_{\varphi}}\right)^{2}+\frac{1}{2} \partial_{\mu} \varphi \partial^{\mu} \varphi-\lambda_{H \varphi}\left(v h+\frac{1}{2} h^{2}\right)\left(v_{\varphi} \varphi+\frac{1}{2} \varphi^{2}\right)$.

Similarly to the fermion DM model, $h$ and $\varphi$ are expressed in terms of the mass eigenstates $H_{1}$ and $H_{2}$ with mixing angle $\alpha$. For the purpose of studying the collider signatures, it would be enough to consider the following simplified VDM with Higgs portal as, ignoring 
the triple and quartic couplings of $H_{1}$ and $H_{2}:^{2}$

$$
\begin{aligned}
\mathcal{L}_{\mathrm{VDM}}= & -\frac{1}{4} V_{\mu \nu} V^{\mu \nu}+\frac{1}{2} m_{V}^{2} V_{\mu} V^{\mu}+\frac{1}{2} \partial_{\mu} \varphi \partial^{\mu} \varphi-\frac{1}{2} \frac{2 m_{V}^{2}}{v_{\varphi}} V_{\mu} V^{\mu}\left(H_{1} \sin \alpha-H_{2} \cos \alpha\right) \\
& -\left(H_{1} \cos \alpha+H_{2} \sin \alpha\right)\left[\sum_{f} \frac{m_{f}}{v_{H}} \bar{f} f-\frac{2 m_{W}^{2}}{v_{H}} W_{\mu}^{+} W^{-\mu}-\frac{m_{Z}^{2}}{v_{H}} Z_{\mu} Z^{\mu}\right] .
\end{aligned}
$$

By defining $g_{V}=2 m_{V} / v_{\varphi}$ and $\lambda_{V}=g_{V} \cos \alpha \sin \alpha$, the scattering amplitude for the process

$$
e^{+}\left(p_{1}\right)+e^{-}\left(p_{2}\right) \rightarrow H_{1} / H_{2}(q)+Z\left(p_{Z}\right) \rightarrow V\left(k_{1}\right)+V\left(k_{2}\right)+Z\left(p_{Z}\right),
$$

is given as

$$
\mathcal{M}_{V}=\mathcal{M}_{h^{*} Z} \cdot \lambda_{V} m_{V}\left[\frac{1}{t-m_{H_{1}}^{2}+i m_{H_{1}} \Gamma_{H_{1}}}-\frac{1}{t-m_{H_{2}}^{2}+i m_{H_{2}} \Gamma_{H_{2}}}\right] \epsilon_{1}^{*}\left(k_{1}\right) \cdot \epsilon_{2}^{*}\left(k_{2}\right) .
$$

The squared amplitude is evaluated to be

$$
\sum\left|\mathcal{M}_{V}\right|^{2}=\left|\mathcal{M}_{h^{*} Z}\right|^{2} \cdot\left(\lambda_{V} m_{V}\right)^{2}\left|\frac{1}{t-m_{H_{1}}^{2}+i m_{H_{1}} \Gamma_{H_{1}}}-\frac{1}{t-m_{H_{2}}^{2}+i m_{H_{2}} \Gamma_{H_{2}}}\right|^{2} \sum\left|\epsilon_{1}^{*} \cdot \epsilon_{2}^{*}\right|^{2},
$$

where the spin-sum of the vector DM wave-functions is calculated as

$$
\sum\left|\epsilon_{1}^{*} \cdot \epsilon_{2}^{*}\right|^{2}=2+\frac{\left(t-2 m_{V}^{2}\right)^{2}}{4 m_{V}^{4}}=\frac{t^{2}}{4 m_{V}^{4}}\left(1-\frac{4 m_{V}^{2}}{t}+\frac{12 m_{V}^{4}}{t^{2}}\right) .
$$

Thus, the $t$ distribution is obtained as

$$
\frac{d \sigma_{V}}{d t}=\frac{1}{2 \pi} \sigma_{h^{*} Z}(s, t) \cdot G_{V}(t)
$$

where

$$
G_{V}(t)=C_{V} \frac{\beta_{V}}{8 \pi} \cdot \frac{\lambda_{V}^{2} t^{2}}{4 m_{V}^{2}}\left(1-\frac{4 m_{V}^{2}}{t}+\frac{12 m_{V}^{4}}{t^{2}}\right) \cdot\left|\frac{1}{t-m_{H_{1}}^{2}+i m_{H_{1}} \Gamma_{H_{1}}}-\frac{1}{t-m_{H_{2}}^{2}+i m_{H_{2}} \Gamma_{H_{2}}}\right|^{2} .
$$

The symmetric factor is $C_{V}=1 / 2$.

\subsection{Comparison of three models}

Before we proceed further, let us make comments on three Higgs portal DM models for scalar, fermion and vector DMs, (2.2), (2.11) and (2.20), respectively. In all the cases, we have imposed $Z_{2}$ symmetry which stabilize DM, $S, \chi$ and $V_{\mu}$. Note that the scalar sectors of these three models are not symmetric: there is only one mediator $(H)$ in the scalar DM case in eq. (2.2), whereas there are two mediators both in the fermion DM case $(H, \phi)$ in eq. (2.11), and in the vector DM cases, $(H, \Phi)$ in eq. (2.20). This is because of the

\footnotetext{
${ }^{2}$ Higgs pair productions will be discussed in the future publication.
} 
gauge invariance and renormalizability. Singlet fermion DM $\psi$ cannot have renormalizable couplings to the SM Higgs field, and one has to introduce a singlet scalar that can couple to $\bar{\chi} \chi$ and mix with the SM Higgs field by $\phi H^{\dagger} H$ and $\phi^{2} H^{\dagger} H$ terms, as in eq. (2.11). Likewise, the vector DM mass cannot be given by hand as in eq. (1.3). It has to be generated, for example, by dark Higgs mechanism by nonzero VEV of $\Phi$ in eq. (2.20). On the other hand, this is not the case for scalar DM, since we can have a gauge invariant and renormalizable $S^{2} H^{\dagger} H$ operator as in eq. (2.2).

In case of fermion or vector DM, these two scalar mediators always appear in combination of eq. $(2.15)$ or $(2.24):^{3}$

$$
\frac{1}{t-m_{H_{1}}^{2}+i m_{H_{1}} \Gamma_{H_{1}}}-\frac{1}{t-m_{H_{2}}^{2}+i m_{H_{2}} \Gamma_{H_{2}}}
$$

whereas for the scalar DM case only the SM Higgs plays the role of mediator,

$$
\frac{1}{t-m_{H_{1}}^{2}+i m_{H_{1}} \Gamma_{H_{1}}} \text {. }
$$

Note that the relative size between two propagators, -1 , in eq. (2.29) is originated from the $\mathrm{SO}(2)$ nature of the rotation matrix from the interaction eigenstates to the mass eigenstates in eq. (2.12).

It may be possible to make three models more symmetric if we introduce additional new fields. For example, we can introduce one more singlet scalar $\phi$ in the scalar DM case with the following additional Lagrangian:

$$
\begin{aligned}
\Delta \mathcal{L}_{\mathrm{SSDM}}= & \frac{1}{2} \partial_{\mu} \phi \partial^{\mu} \phi-\frac{1}{2} m_{\phi}^{2} \phi^{2}-\mu_{\phi}^{\prime 3} \phi-\frac{\lambda_{\phi}}{4} \phi^{4} \\
& -\mu_{\phi H} \phi H^{\dagger} H-\frac{1}{2} \mu_{\phi S} \phi S^{2}-\frac{1}{2} \lambda_{\phi H} \phi^{2} H^{\dagger} H-\frac{1}{4} \phi^{2} S^{2} .
\end{aligned}
$$

Then there will be two scalar mediators, $H_{1}$ and $H_{2}$, but the relative sign and magnitudes of these two contributions to the processes we consider would be completely free, and is not fixed to be -1 as in eq. (2.31). This is because both the singlet scalar $\phi$ and the SM Higgs $H$ can have renormalizable couplings to scalar DM $S$. We do not consider this case further, since it is not minimal in terms of the number of degrees of freedom.

This difference in the number of force mediators in the scalar DM and in the fermion/vector DM cases will generate the difference in the various differential distributions studied (see figure 1, for example). And this difference will make the high- $t$ behaviors of the amplitudes very different, see eqs. (3.1)-(3.5) and the discussions.

\section{How to distinguish 3 cases at the ILC?}

As we have evaluated in the previous section, the $t\left(=M_{D D}^{2}\right)$ spectrum is given by

$$
\frac{d \sigma}{d t} \propto F(s, t) \times G_{D}(t)
$$

\footnotetext{
${ }^{3}$ We assume that $H_{1}$ is the $125 \mathrm{GeV}$ scalar boson observed at the LHC.
} 
where the $t$-dependent form factor $G_{D}(t)$ is given by

$\operatorname{SSDM}: G_{S}(t) \sim \beta_{D} \frac{1}{\left(t-m_{H}^{2}\right)^{2}+m_{H}^{2} \Gamma_{H}^{2}}$,

$\operatorname{SFDM~:~} \quad G_{\chi}(t) \sim \beta_{D}^{3}\left|\frac{1}{t-m_{H_{1}}^{2}+i m_{H_{1}} \Gamma_{H_{1}}}-\frac{1}{t-m_{H_{2}}^{2}+i m_{H_{2}} \Gamma_{H_{2}}}\right|^{2}\left(t-4 m_{\chi}^{2}\right)$

$\rightarrow\left|\frac{1}{t^{2}}\right|^{2} \times t \sim \frac{1}{t^{3}}\left(\right.$ as $t \rightarrow \infty$ for fixed $m_{H_{1}}$ and $\left.m_{H_{2}}\right)$,

$\mathrm{VDM}: \quad G_{V}(t) \sim \beta_{D}\left|\frac{1}{t-m_{H_{1}}^{2}+i m_{H_{1}} \Gamma_{H_{1}}}-\frac{1}{t-m_{H_{2}}^{2}+i m_{H_{2}} \Gamma_{H_{2}}}\right|^{2}\left[2+\frac{\left(t-2 m_{V}^{2}\right)^{2}}{4 m_{V}^{4}}\right]$ $\rightarrow\left|\frac{1}{t^{2}}\right|^{2} \times t^{2} \sim \frac{1}{t^{2}}\left(\right.$ as $t \rightarrow \infty$ for fixed $m_{H_{1}}$ and $\left.m_{H_{2}}\right)$.

The signal distribution arises at $t=\left(2 m_{D}\right)^{2}$, thus by measuring the threshold of the signal excess, the DM mass can be directly determined. In addition, the threshold slope of the signal excess depends on the spin of DM; for scalar and vector DM models it behaves as $\propto \beta_{D}$, while for the fermion DM model it behave as $\beta_{D}^{3}$. This is also useful to distinguish the spin of DM by a kinematical method [46].

The $t$ distribution in the high- $t$ region is sensitive to the unitarity of the models. If we ignore the 2nd Higgs propagator and identify $m_{H_{1}}=m_{H}$ (the discovered Higgs boson), we would have

$$
\begin{aligned}
\operatorname{SFDM}: \quad G_{\chi}(t) & \sim \beta_{D}^{3} \frac{1}{\left(t-m_{H}^{2}\right)^{2}+m_{H}^{2} \Gamma_{H}^{2}}\left(t-4 m_{\chi}^{2}\right) \\
& \rightarrow \frac{1}{t}(\operatorname{as~} t \rightarrow \infty) \\
\mathrm{VDM}: \quad G_{V}(t) & \sim \beta_{D} \frac{1}{\left(t-m_{H}^{2}\right)^{2}+m_{H}^{2} \Gamma_{H}^{2}}\left[2+\frac{\left(t-2 m_{V}^{2}\right)^{2}}{4 m_{V}^{4}}\right] \\
& \rightarrow \text { constant }(\operatorname{as} t \rightarrow \infty) .
\end{aligned}
$$

These results indicate the violation of unitarity in the total cross section at large $s$ from large- $t$ region, and the EFT descriptions based on (1.2) and (1.3) would become unreliable. Note that ignoring the propagator of the 2nd Higgs would be justified if $m_{H_{2}} \gg \sqrt{s}$. On the other hand, in the UV-completed approach, the distribution behaves well convergent at high- $t$.

Therefore, one would be able to determine the type of DM by observing the shape of the signal distribution. Having enough number of bins and data, we can test by $\chi^{2}$ minimization to determine whether the observed $\mathbb{E}_{T}$ distribution follows that of scalar, fermion or vector DM with Higgs portal. Note that this procedure is possible at the ILC, and not at LHC, since at the ILC the CM energy $\sqrt{s}$ is fixed so that one can factor out the phase-space factor. On the other hand, at hadron colliders, the parton-level CM energy $\sqrt{\hat{s}}$ is not fixed so that we cannot factor out the phase-space factor in an unambiguous manner. Note that for scalar DM, $G_{S}(t)$ is completely fixed by the SM Higgs propagator, and there is no free parameter except $m_{D}$. Therefore it would be straightforward to check 
if the observed $\mathbb{E}_{T}$ distribution can be fitted by the SM Higgs propagator or not. For the SFDM or VDM models, the fitting would be more complicated, since in this case, there are 4 parameters: namely,

$\sin \alpha, m_{H_{2}}, \quad \Gamma_{H_{2}}, m_{\mathrm{D}}$.

Note that we have to regard $\Gamma_{H_{2}}$ and $\sin \alpha$ independently, since $H_{2} \rightarrow H_{1} H_{1}$ can be newly open, which calls a new parameter that could be traded with $\Gamma_{H_{2}}$. With these 4 parameters, we can fit the $\mathbb{E}_{T}$ spectrum or the recoil-mass spectrum, $M_{\text {rec }}=\sqrt{t}$, and determine the physical parameters in the SFDM or VDM models.

In figure 1 , the normalized recoil-mass distribution $1 / \sigma \cdot d \sigma / d M_{\text {rec }}$ is evaluated for the scalar, fermion and vector DM models for various sets of $\left(m_{D}, m_{H_{2}}\right)$ at $\sqrt{s}=500 \mathrm{GeV}$. $\Gamma_{H_{2}}=0.1 \mathrm{GeV}$ is used for simplicity. The characteristic threshold behavior as well as the large recoil-mass tail can be understood by the analytically-calculated form factors given in the previous section. For $2 m_{D} \leq m_{H_{2}} \leq \sqrt{s}-m_{Z}$, on-shell $H_{2}$ can be produced and subsequently decay into a pair of the DM particle. In such cases, the recoil mass distribution shows a sharp peak at $M_{\text {rec }}=m_{H_{2}}$, and no difference can be observed between the fermion DM and vector DM models. In addition, if $m_{D} \leq m_{H_{1}} / 2$, the recoil mass distribution shows another peak at $M_{\text {rec }}=m_{H_{1}}$ whose strength is expected to be smaller than the peak at $m_{H_{2}}$ because of the constraints so far [see section 4.1]. Spin discrimination is still difficult since it behaves as a sharp peak for each DM model. For $2 m_{D} \geq m_{H_{2}}$ or $m_{H_{2}} \geq \sqrt{s}-m_{Z}$, no sharp peak can be observed, because on-shell $H_{2}$ cannot be produced because of the limited collision energy or because the on-shell $H_{2}$ cannot decay into a DM pair, so that the DM pair is produced through the off-shell $H_{1}$ and $H_{2}$. The recoil-mass distributions then behave as smooth curves depending on the DM model as well as the masses of DM and $\mathrm{H}_{2}$. Thus, by measuring the shape of the distribution, one can determine the type of DM and its mass, as well as the mass of the second Higgs portal to DM.

\section{Parameter constraints}

In this section, we discuss searches for the Higgs portal DM models at the future lepton colliders. Depending on the masses of DM and the second Higgs boson in the fermion and vector DM models, the search strategy at colliders can be different. In figure 2, we divide the parameter space in the $\left(m_{H_{2}}-m_{D}\right)$ plane in terms of the plausible collider signature to search for in each parameter region in the fermion and vector DM models. In the scalar DM model, because of the absence of $H_{2}$, the parameter region can be simply divided by $m_{D}<m_{H_{1}} / 2$ or $m_{D}>m_{H_{1}} / 2$, namely whether the observed Higgs boson can decay into the DM pair or not. In the following subsections, we discuss collider signals of DM production in each region of the parameter space, then further discuss the method to distinguish models, and to determine the model parameters.

\section{$4.1 m_{D} \leq m_{H_{1}} / 2$}

In the case of $m_{D} \leq m_{H_{1}} / 2$, a DM pair can be produced in the decay of $H_{1}$. This can be seen as an invisible decay of the Higgs boson. The limit on the branching ratio of the invisible de- 

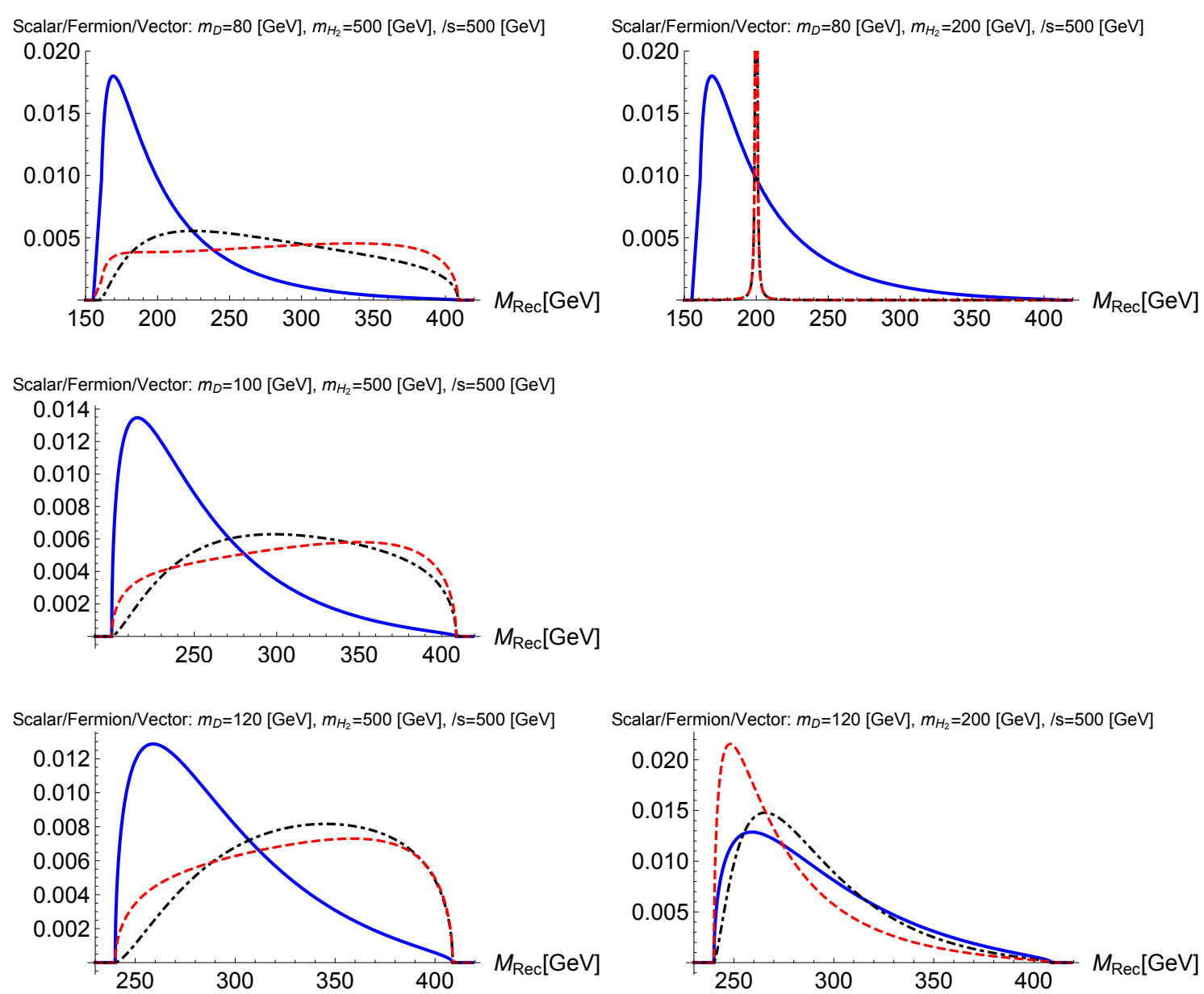

Figure 1. Normalized recoil-mass distribution in $e^{+} e^{-} \rightarrow Z H_{1}\left(/ H_{2}^{(*)}\right) \rightarrow Z D D$ at $\sqrt{s}=500 \mathrm{GeV}$. Blue: scalar DM, Black: fermion DM, Red: vector DM.

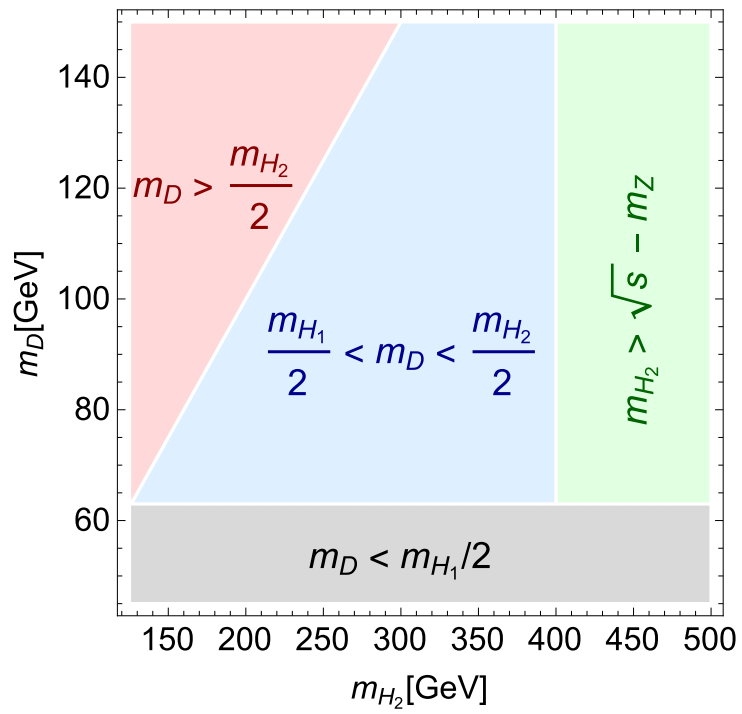

Figure 2. Dividing parameter space in the $\left(m_{H_{2}}-m_{D}\right)$ plane by the plausible collider signature in the fermion and vector DM models. In the scalar DM model, $H_{2}$ is absent. 
cay has been obtained as $\mathcal{B}(h \rightarrow$ inv. $) \lesssim 0.25$ at the LHC [47-50]. We see how the model parameters can be constrained by this measurement. In the scalar DM model, the partial decay width for $H_{1} \rightarrow S S$ is proportional to $\lambda_{H S}^{2}$. By denoting $\Gamma\left(H_{1} \rightarrow S S\right)=\lambda_{H S}^{2} \Gamma_{0}$, the experimental constraint of $\mathcal{B}(h \rightarrow$ inv. $)<\left.\mathcal{B}(h \rightarrow$ inv. $)\right|_{\text {exp. }} \equiv X$, we obtain the limit on $\lambda_{\text {HS }}$ as

$$
\lambda_{H S}^{2}<\frac{X}{1-X} \frac{\Gamma_{h}^{\mathrm{SM}}}{\Gamma_{0}} .
$$

For the fermion and vector DM models, the partial decay width for $H_{1} \rightarrow \chi \bar{\chi}(V V)$ is proportional to $y_{\chi}^{2} s_{\alpha}^{2}\left(\lambda_{V}^{2} s_{\alpha}^{2}\right)$. On the other hand, the partial widths for the decay into SM particles are all suppressed by $c_{\alpha}^{2}$. By writing $\Gamma\left(H_{1} \rightarrow F \bar{F}\right)=y_{\chi}^{2} s_{\alpha}^{2} \Gamma_{D}\left[\Gamma\left(H_{1} \rightarrow F \bar{F}\right)=\right.$ $\left.g_{V}^{2} s_{\alpha}^{2} \Gamma_{D}\right]$, the constraint on the branching ratio $\mathcal{B}(h \rightarrow$ inv. $)<X$ gives

$$
y_{\chi}^{2}\left[g_{V}^{2}\right] \cdot t_{\alpha}^{2}<\frac{X}{1-X} \frac{\Gamma_{\mathrm{SM}}}{\Gamma_{D}} .
$$

Here, $t_{\alpha}=\tan \alpha$. We evaluate the current limit by the LHC Run-I measurement, $\mathcal{B}\left(h_{\mathrm{SM}} \rightarrow\right.$ inv. $) \leq 0.25$ [48], and also the accessible limits at future experiments, $\mathcal{B}\left(h_{\mathrm{SM}} \rightarrow\right.$ inv. $) \leq$ 0.0065 at the ILC $500 \mathrm{GeV}$ with $500 \mathrm{fb}^{-1}$, and $\leq 0.0032$ with $1600 \mathrm{fb}^{-1}$ [13]. The limits are obtained on $\lambda_{H S}, y_{\chi} t_{\alpha}$ and $g_{V} t_{\alpha}$ in the scalar, fermion and vector DM models, respectively. For the fermion (vector) DM model, the constraint on the coupling $y_{\chi}\left(g_{V}\right)$ becomes weak for small $s_{\alpha}$. The upper bound on $s_{\alpha}$ has been obtained by measuring the signal strength of Higgs-gauge-gauge coupling $\kappa_{V}$ at the LHC Run-I, which is equal to $c_{\alpha}$ in our models. Current limit is about $\kappa_{V} \gtrsim 0.85$ at the $68 \%$ C.L. [42-44], thus $s_{\alpha} \lesssim 0.53$. For the reference, in the cases of $c_{\alpha}=0.9,0.95$ and $0.99, t_{\alpha}$ are $\simeq 0.48,0.33$ and 0.14 , respectively.

In the top-left, top-right and bottom panels in figure 3, current and future limits on the parameters in the scalar, fermion and vector DM models with $m_{D} \leq m_{H_{1}} / 2$ are plotted as a function of $m_{D}$, respectively. In the scalar DM model, $\lambda_{H S}$ is constrained to be $\gtrsim 0.01$ for $m_{D} \lesssim m_{H_{1}} / 2$. At the future ILC measurements, $\lambda_{H S} \gtrsim 0.001$ will be explored. In the fermion and vector DM models, the constraints on $y_{\chi} t_{\alpha}\left(g_{V} t_{\alpha}\right)$ are $\gtrsim 0.01$ by current LHC measurement, and will be $\gtrsim 0.001$ by future ILC measurements. By observing non-zero $s_{\alpha}$ in future measurements, limits on the Higgs-DM-DM coupling can be derived.

\section{$4.2 m_{D} \geq m_{H_{1}} / 2$}

In the scalar DM model with $m_{D} \geq m_{H_{1}} / 2$, a DM pair is produced through the off-shell $H_{1}$. The collider signal for this case can be an excess in events with a $Z$-boson plus missing energy with a large recoil-mass, $M_{\text {rec. }} \geq 2 m_{D}$. We consider muonic and hadronic decays of $Z$-boson whose branching ratios are $\mathcal{B}\left[Z \rightarrow \mu^{+} \mu^{-}\right] \simeq 3.4 \%$ and $\mathcal{B}[Z \rightarrow j j] 70 \%$, respectively [51]. The dimuon channel has limited number of events, but is promised to be observed because of the clear signal and fine momentum-resolution. The signal in dijet channel has large number of events because of the large branching ratio, but may be affected by large reducible background events and less momentum resolution for jet measurements.

In the fermion and vector DM models, another Higgs boson $\mathrm{H}_{2}$ has been introduced. If $H_{2}$ can be produced on-shell, and its decay branching ratio into a DM pair is sizable, we expect the invisible decay of $H_{2}$ as a signal of the DM production. This can be investigated by searching for another peak in the recoil mass distribution in events with $Z$-boson plus 

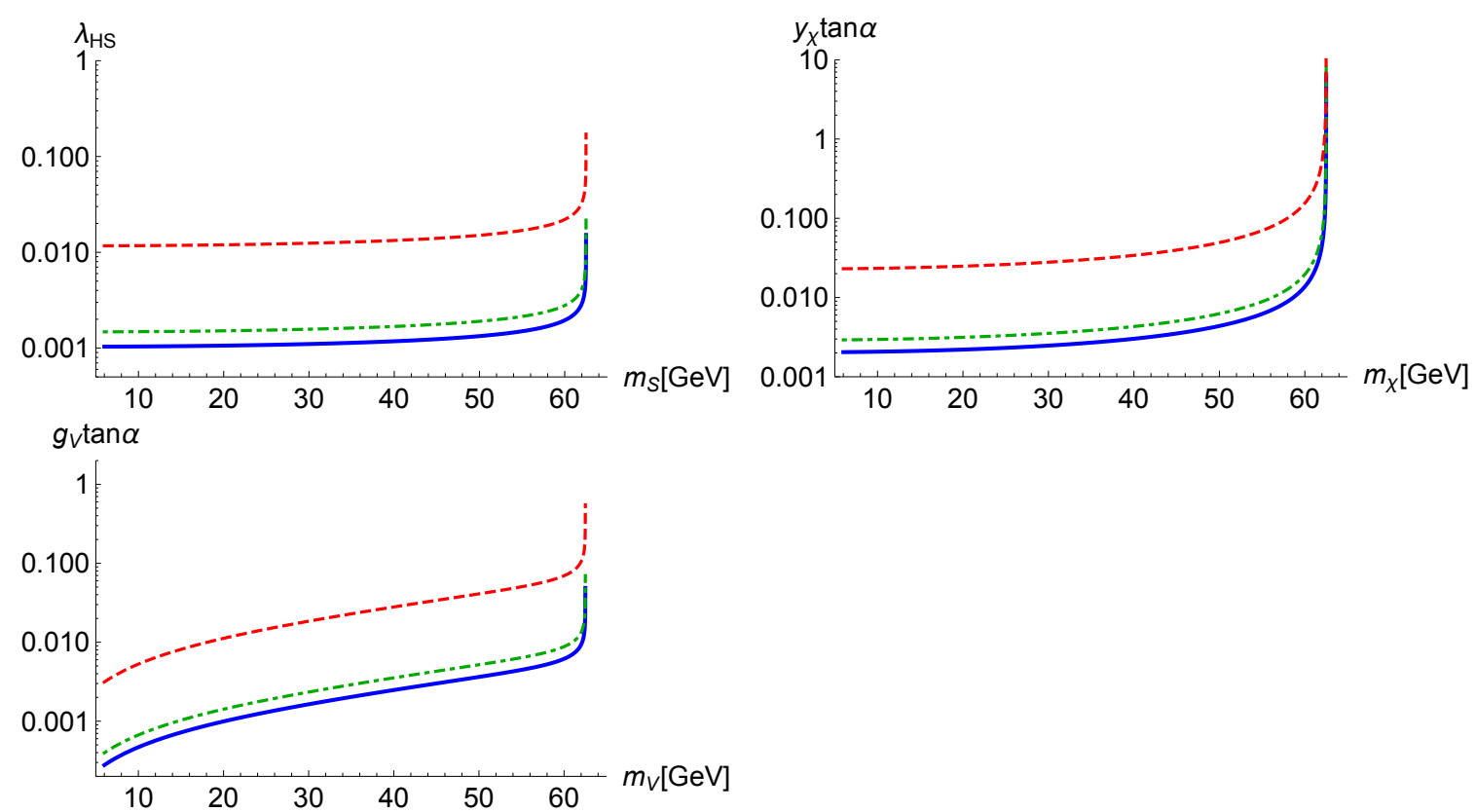

Figure 3. Current and future limits on the parameters in the scalar, fermion and vector DM models with $m_{D} \leq m_{H_{1}} / 2$. Constraints on the branching ratio of the Higgs invisible decay at the LHC Run-I (red, dashed), ILC $500 \mathrm{GeV}$ with $500 \mathrm{fb}^{-1}$ (green dot-dashed), and $1600 \mathrm{fb}^{-1}$ (blue solid) are considered.

missing energy. On the other hand, even if $H_{2}$ cannot be produced on-shell or $\mathrm{H}_{2}$ cannot decay into a DM pair by kinematical reasons, a DM pair can be produced through the off-shell propagation of $H_{1}$ and $H_{2}$. The collider signal for this case would be an excess in a relatively wide region in the recoil-mass distribution for the events with $Z$-boson plus missing energy. As we discussed, the propagators of $H_{1}$ and $H_{2}$ are $\propto \mid\left(t-m_{H_{1}}^{2}+\right.$ $\left.i m_{H_{1}} \Gamma_{H_{1}}\right)^{-1}-\left.\left(t-m_{H_{2}}^{2}+i m_{H_{2}} \Gamma_{H_{2}}\right)^{-1}\right|^{2}$. Thus, for $m_{H_{1}}^{2}<t<m_{H_{2}}^{2}$, this gives a constructive interference. We emphasize here again that the mass, spin of DM as well as the mass of another Higgs boson can be explored by studying the shape of the recoil-mass distribution.

\subsubsection{Scalar DM, $m_{D} \geq m_{H_{2}} / 2$ or $m_{H_{2}} \geq \sqrt{s}-m_{Z}$ cases}

In the cases of the scalar DM model, and the fermion and vector DM models with $m_{D} \geq$ $m_{H_{2}} / 2$ or $m_{H_{2}} \geq \sqrt{s}-m_{Z}$, a DM pair is produced via $e^{+} e^{-} \rightarrow Z H_{1}^{*}\left(/ H_{2}^{*}\right) \rightarrow Z D D$. The amplitude is proportional to $\lambda_{H S}, \lambda_{\chi}=y_{\chi} s_{\alpha} c_{\alpha}$ and $\lambda_{V}=g_{V} s_{\alpha} c_{\alpha}$ in the scalar, fermion and vector DM models, respectively. Therefore by observing the $Z$-boson plus missing energy events at large $M_{\text {rec }}\left(\geq 2 m_{D}\right)$, these parameters can be determined or constrained. We study the feasibility of detecting this process at the ILC by a simple MC simulation using MadGraph version 5 MadGraph5 [52]. The signal of the process can be a reconstructed $Z$-boson plus missing energy;

$$
e^{+} e^{-} \rightarrow Z H_{1}^{*}\left(/ H_{2}^{*}\right) \rightarrow Z D D \rightarrow\left(j j \text { or } \mu^{+} \mu^{-}\right)+\not e,
$$

where we consider the hadronic and muonic decays of the $Z$-boson. Major SM background events in the hadronic channel come from $e^{+} e^{-} \rightarrow Z \nu_{\ell} \bar{\nu}_{\ell} \rightarrow j j \nu_{\ell} \bar{\nu}_{\ell}$ with $\ell=e, \mu, \tau$. On 
the other hand, those in the muonic channel come from $e^{+} e^{-} \rightarrow \mu^{+} \mu^{-} \nu_{\ell} \bar{\nu}_{\ell}$ with $\ell=e, \mu, \tau$. The total cross sections for these background processes in the dimuon and dijet channels are calculated to be $107 \mathrm{fb}$ and $345 \mathrm{fb}$, respectively, at $\sqrt{s}=500 \mathrm{GeV}$.

To reduce the SM background, we consider following kinematical cuts;

$$
\begin{aligned}
p_{T}^{Z} & \geq 100 \mathrm{GeV} \\
\left|\eta^{Z}\right| & \leq 1 \\
2 m_{D} & \leq M_{\mathrm{rec}} \leq 2 m_{D}+50[\mathrm{GeV}] .
\end{aligned}
$$

We simulate the signal and background events by using MadGraph5 at the parton level, and estimate the efficiencies by these cuts. In table 1, we summarize the total cross-section of the signal process divided by $\lambda_{H S}^{2}$, the efficiencies for the signal and background events by kinematical cuts as a function of the DM mass $m_{S}$ for $\sqrt{s}=500 \mathrm{GeV}$. The DM mass is examined from $80 \mathrm{GeV}$ to $160 \mathrm{GeV}$. By these kinematical cuts, about $60 \%$ to $20 \%$ of signal events survive depending on the mass of DM, while background events are suppressed to $\mathcal{O}(0.1 \%)$ level in the dimuon channel and $\mathcal{O}(1 \%)$ level in the dijet channel.

We estimate the significance of detecting the excess in events with $Z$-boson plus missing energy by

$$
S=\frac{\sigma_{Z D D} \mathcal{B}\left(Z \rightarrow \mu^{+} \mu^{-} / j j\right) \epsilon_{S} \mathcal{L}}{\sqrt{\sigma_{\mathrm{BG}} \epsilon_{B} \mathcal{L}}} .
$$

We say $S \geq 5$ is required to discover signal events. Because the cross section scales with $\lambda_{H S}^{2}$, we can evaluate the lower limit of $\lambda_{H S}\left(\lambda_{H S}^{\min }\right)$ to be detected by a certain accumulated luminosity for each $m_{S}$. In table 1 , our estimations for $\lambda_{H S}^{\min }$ are also listed assuming $\mathcal{L}=500 \mathrm{fb}^{-1}$ and $1600 \mathrm{fb}^{-1}$. In figure 4 , we plot $\lambda_{H S}^{\min }$ in the dimuon channel (red lines) and the dijet channel (blue lines) for $\mathcal{L}=500 \mathrm{fb}^{-1}$ (dashed lines) and $1600 \mathrm{fb}^{-1}$ (solid lines). We find that in the future ILC experiment with $\sqrt{s}=500 \mathrm{GeV}, \lambda_{H S} \leq 1$ can be surveyed only for light DM cases $\left(m_{S} \lesssim 80 \mathrm{GeV}\right)$, and only $\lambda_{H S} \geq 10$ can be surveyed for heavier DM cases like $m_{S} \geq 150 \mathrm{GeV}$.

In the fermion and vector DM models, the production cross section depends on $m_{H_{2}}$ as well. We consider $m_{H_{2}}=500 \mathrm{GeV}$ and $m_{H_{2}}=200 \mathrm{GeV}$ for example. In the large $m_{H_{2}}$ limit, the diagram with $H_{2}$ propagator decouples, and the collider phenomenology becomes the same as that for the simple extension of the SM by adding only fermion or vector DM. ${ }^{4}$ In table 2, we summarize the analysis for the fermion DM model with $m_{H_{2}}=500 \mathrm{GeV}$. The signal cross section, efficiencies, and the lower limit of $\lambda_{\chi}=y_{\chi} c_{\alpha} s_{\alpha}$ to be detected in dimuon and dijet channels by $5 \sigma$ C.L. assuming $\mathcal{L}=500$ and $1600 \mathrm{fb}^{-1}$ are summarized. In the

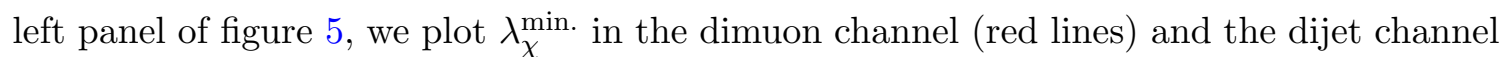
(blue lines) for $\mathcal{L}=500 \mathrm{fb}^{-1}$ (dashed lines) and $1600 \mathrm{fb}^{-1}$ (solid lines). We perform the same analysis for $m_{H_{2}}=200 \mathrm{GeV}$, and the results are shown in the right panel of figure 5 .

In table 3 and figure 6 , the same analyses for the vector DM model are summarized, where $\lambda_{V}=g_{V} c_{\alpha} s_{\alpha}$ is constrained by the measurement.

\footnotetext{
${ }^{4}$ Modifying the SM Higgs boson couplings by $\kappa_{V}=\kappa_{F}=\cos \alpha$ is an important consequence of making the Higgs portal DM models SM gauge invariant and unitary [32, 36].
} 


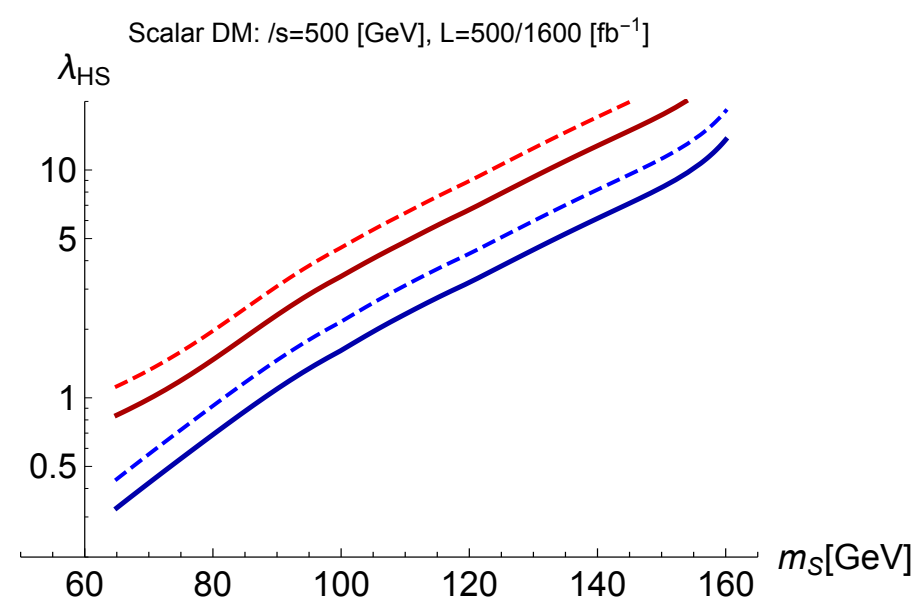

Figure 4. Contour plots for the discovery potential at 95\% C.L. in $e^{+} e^{-} \rightarrow Z D D$ searches at the ILC with $\sqrt{s}=500 \mathrm{GeV}$ and $\mathcal{L}=500$ (dashed), 1600 (solid) [ $\left.\mathrm{fb}^{-1}\right]$. Red contours are the limits by using the dimuon channel of $Z$-boson decay, and blue contours are the limits by using the dijet channel.
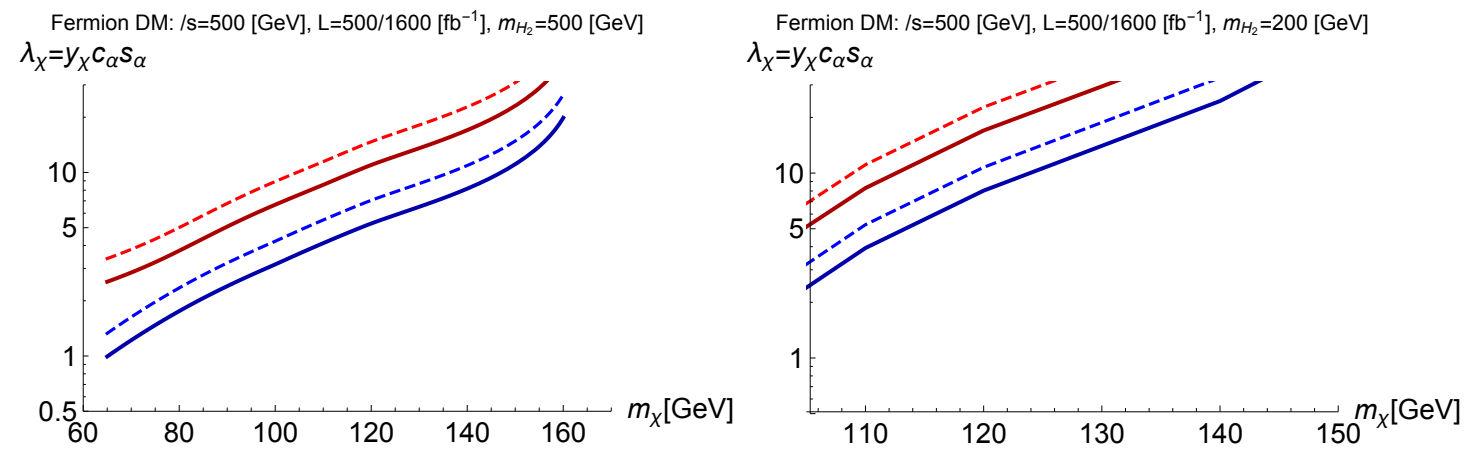

Figure 5. The same figure as figure 4, but for the fermion DM model with $m_{H_{2}}=500 \mathrm{GeV}$ [left] and $200 \mathrm{GeV}$.
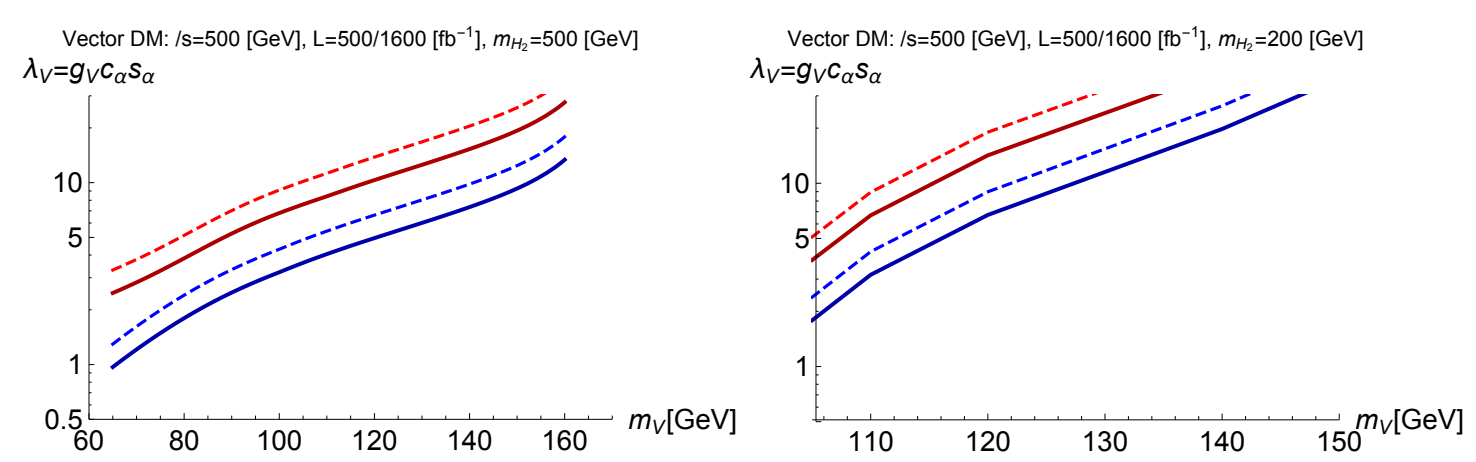

Figure 6. The same figure as figure 4 , but for the vector DM model with $m_{H_{2}}=500 \mathrm{GeV}$ [left] and $200 \mathrm{GeV}$. 


\begin{tabular}{|l|ccccccc|}
\hline \multicolumn{7}{|c|}{ Scalar DM Model, $\sqrt{s}=500 \mathrm{GeV}$} \\
$m_{S}[\mathrm{GeV}]$ & 80 & 90 & 100 & 110 & 120 & 140 & 160 \\
\hline$\sigma_{Z S S} / \lambda_{H S}^{2}[\mathrm{fb}]$ & 1.37 & 0.69 & 0.38 & 0.22 & 0.13 & 0.046 & 0.014 \\
$\epsilon_{S}[\%]$ & 56 & 51 & 48 & 47 & 48 & 45 & 23 \\
\hline \multicolumn{1}{|c}{$Z \rightarrow \mu^{+} \mu^{-}$} & & & & & & & \\
$\epsilon_{B}[\%]$ & 0.19 & 0.24 & 0.32 & 0.41 & 0.55 & 0.80 & 0.44 \\
$\lambda_{H S}^{\min .}\left(\mathcal{L}=500 \mathrm{fb}^{-1}\right)$ & 1.97 & 3.09 & 4.56 & 6.47 & 8.93 & $(17.1)$ & $(37.5)$ \\
$\lambda_{H S}^{\min }\left(\mathcal{L}=1600 \mathrm{fb}^{-1}\right)$ & 1.47 & 2.31 & 3.41 & 4.84 & 6.68 & $(12.8)$ & $(28.1)$ \\
\hline \multicolumn{1}{|c}{$Z \rightarrow j j$} & & & & & & & \\
$\epsilon_{B}[\%]$ & 1.2 & 1.6 & 2.1 & 2.9 & 3.8 & 5.6 & 3.2 \\
$\lambda_{H S}^{\min .}\left(\mathcal{L}=500 \mathrm{fb}^{-1}\right)$ & 0.922 & 1.46 & 2.16 & 3.11 & 4.29 & 8.20 & $(18.2)$ \\
$\lambda_{H S}^{\min .}\left(\mathcal{L}=1600 \mathrm{fb}^{-1}\right)$ & 0.689 & 1.09 & 1.61 & 2.33 & 3.21 & 6.13 & $(13.6)$ \\
\hline
\end{tabular}

Table 1. Signal and background efficiencies for detecting $e^{+} e^{-} \rightarrow Z H_{1} \rightarrow Z D D$ process at the ILC. Prospects for parameter constraints are also shown assuming $\mathcal{L}=500 \mathrm{fb}^{-1}$ and $1600 \mathrm{fb}^{-1}$. Limits on the couplings larger than $4 \pi$ are presented within parentheses.

\begin{tabular}{|l|ccccccc|}
\hline \multicolumn{7}{|c|}{ Fermion DM Model, $\sqrt{s}=500 \mathrm{GeV}, m_{H_{2}}=500 \mathrm{GeV}$} \\
$m_{\chi}[\mathrm{GeV}]$ & 80 & 90 & 100 & 110 & 120 & 140 & 160 \\
\hline $\begin{array}{l}\sigma_{Z F F} / \lambda_{\chi}^{2}[\mathrm{fb}] \\
\epsilon_{S}[\%]\end{array}$ & 0.76 & 0.53 & 0.37 & 0.26 & 0.18 & 0.077 & 0.025 \\
\hline \multicolumn{1}{|c}{$Z \rightarrow \mu^{+} \mu^{-}$} & 15 & 13 & 13 & 13 & 13 & 15 & 6 \\
$\epsilon_{B}[\%]$ & 0.19 & 0.24 & 0.32 & 0.41 & 0.55 & 0.80 & 0.44 \\
$\lambda_{\chi}^{\min .}\left(\mathcal{L}=500 \mathrm{fb}^{-1}\right)$ & 5.01 & 6.78 & 8.92 & 11.4 & $(14.7)$ & $(22.7)$ & $(54.8)$ \\
$\lambda_{\chi}^{\min }\left(\mathcal{L}=1600 \mathrm{fb}^{-1}\right)$ & 3.75 & 5.07 & 6.67 & 8.56 & 11.0 & $(17.0)$ & $(41.0)$ \\
\hline \multicolumn{1}{|c}{$Z \rightarrow j j$} & & & & & & & \\
$\epsilon_{B}[\%]$ & 1.2 & 1.6 & 2.1 & 2.9 & 3.8 & 5.6 & 3.2 \\
$\lambda_{\chi}^{\min .}\left(\mathcal{L}=500 \mathrm{fb}^{-1}\right)$ & 2.35 & 3.21 & 4.22 & 5.51 & 7.06 & 10.9 & $(26.5)$ \\
$\lambda_{\chi}^{\min .}\left(\mathcal{L}=1600 \mathrm{fb}^{-1}\right)$ & 1.76 & 2.40 & 3.16 & 4.12 & 5.28 & 8.16 & $(19.8)$ \\
\hline
\end{tabular}

Table 2. The same as table 1, but for the fermion DM case.

\subsubsection{Fermion and vector DM models with $m_{D} \leq m_{H_{2}} / 2$}

In the case with $m_{D} \leq m_{H_{2}} / 2$ in the fermion and vector DM models, if $H_{2}$ is light enough to be produced, DM can be searched for as an invisible decay of $H_{2}$, since the coupling of the dark matter to the another Higgs boson is expected to be sizable in the Higgs portal scenario. Here, we study the production of $H_{2}$ in $e^{+} e^{-} \rightarrow Z H_{2}$ where $Z$ decays into $\mu^{+} \mu^{-}$or $j j$, and $H_{2}$ decays into $D D$. This signal can be a part of the inclusive $H_{2}$ search in $e^{+} e^{-} \rightarrow Z X$ process where $H_{2}$ can be detected by a new peak in the recoil mass distribution at $M_{\text {rec. }} \simeq m_{H_{2}}$. 


\begin{tabular}{|c|c|c|c|c|c|c|c|}
\hline \multicolumn{8}{|c|}{ Vector DM Model, $\sqrt{s}=500 \mathrm{GeV}, m_{H_{2}}=500 \mathrm{GeV}$} \\
\hline$m_{V}[\mathrm{GeV}]$ & 80 & 90 & 100 & 110 & 120 & 140 & 160 \\
\hline$\sigma_{Z V V} / \lambda_{V}^{2}[\mathrm{fb}]$ & 0.74 & 0.47 & 0.31 & 0.21 & 0.14 & 0.064 & 0.026 \\
\hline$\epsilon_{S}[\%]$ & 15 & 14 & 15 & 16 & 19 & 23 & 13 \\
\hline$Z \rightarrow \mu^{+} \mu^{-}$ & & & & & & & \\
\hline$\epsilon_{B}[\%]$ & 0.19 & 0.24 & 0.32 & 0.41 & 0.55 & 0.80 & 0.44 \\
\hline$\lambda_{V}^{\min .}\left(\mathcal{L}=500 \mathrm{fb}^{-1}\right)$ & 5.13 & 7.02 & 9.10 & 11.2 & $(13.8)$ & $(20.4)$ & $(37.0)$ \\
\hline$\lambda_{V}^{\min }\left(\mathcal{L}=1600 \mathrm{fb}^{-1}\right)$ & 3.84 & 5.25 & 6.80 & 8.41 & 10.3 & $(15.3)$ & $(27.6)$ \\
\hline$Z \rightarrow j j$ & & & & & & & \\
\hline$\epsilon_{B}[\%]$ & 1.2 & 1.6 & 2.1 & 2.9 & 3.8 & 5.6 & 3.2 \\
\hline$\lambda_{V}^{\min .}\left(\mathcal{L}=500 \mathrm{fb}^{-1}\right)$ & 2.40 & 3.33 & 4.30 & 5.41 & 6.63 & 9.82 & $(17.9)$ \\
\hline$\lambda_{V}^{\min .}\left(\mathcal{L}=1600 \mathrm{fb}^{-1}\right)$ & 1.80 & 2.49 & 3.22 & 4.05 & 4.96 & 7.34 & $(13.4)$ \\
\hline
\end{tabular}

Table 3. The same as table 1 , but for the vector DM case.

We also perform a simulation analysis to study to what extent the signal can be detected at future lepton colliders with $\sqrt{s}=500 \mathrm{GeV}$. The total event rate is estimated by $\sigma\left(Z H_{2}\right) \mathcal{B}\left(H_{2} \rightarrow D D\right)$ where $\sigma\left(Z H_{2}\right)$ is proportional to $s_{\alpha}^{2}$. We consider a scenario where $\mathcal{B}\left(H_{2} \rightarrow D D\right)$ is large. For simplicity, we take $\mathcal{B}\left(H_{2} \rightarrow D D\right)=1$. Then, $s_{\alpha}$ is determined or constrained by the experimental measurement. To enhance the signal significance in the presence of background events, we apply the same kinematical cuts in eqs. (4.4) but the cut on $M_{\text {rec. }}$ is replaced by

$$
\left|M_{\mathrm{rec}}-m_{H_{2}}\right| \leq 10[\mathrm{GeV}],
$$

because of the sharp peak in the signal events. In table 4 , we summarize the signal crosssection, signal and background efficiencies by cuts, and the lower limits of $s_{\alpha}$ to be observed at $5 \sigma$ C.L. by using dimuon or dijet decays of $Z$ boson and by assuming $\mathcal{L}=500$ or $1600 \mathrm{fb}^{-1}$. We find that $s_{\alpha} \simeq 0.1-0.2$ can be investigated for $m_{H_{2}}=150-300 \mathrm{GeV}$ under the assumption of $\mathcal{B}\left[H_{2} \rightarrow D D\right]=1$. In figure 7 , the lower limits of $s_{\alpha}$ to be observed are plotted as a function of $m_{H_{2}}$.

We make some comments on this analysis. First, because we have assumed $\mathcal{B}\left[\mathrm{H}_{2} \rightarrow\right.$ $D D]=1$, the signal sensitivity may be maximized. For smaller $\mathcal{B}\left[H_{2} \rightarrow D D\right]$, the number of signal events is decreased and the sensitivity on $s_{\alpha}$ would be weakened. In addition, the analysis does not depend on $m_{D}$, except demanding $m_{D} \leq m_{H_{2}} / 2$. In the actual models, branching ratios of $H_{2}$ should be predicted and calculated as a function of $s_{\alpha}, m_{H_{2}}, m_{D}$ and $\Gamma\left(H_{2} \rightarrow H_{1} H_{1}\right)$. The last quantity can be replaced by model parameters in the Higgs potential in the model. More concrete analysis may be required to determine the model parameters in general situations. Second, there is no distinction between the fermion DM model and vector DM model in this measurement, since the recoil mass distribution behaves just a sharp peak. Events in the off-peak region may be useful to distinguish the models based on kinematics, if enough number of events are collected. 


\begin{tabular}{|l|ccccc|}
\hline \multicolumn{5}{|c|}{$e^{+} e^{-} \rightarrow Z H_{2}(\rightarrow D D), \sqrt{s}=500 \mathrm{GeV}, \mathcal{B}\left[H_{2} \rightarrow D D\right]=1$} \\
$m_{H_{2}}[\mathrm{GeV}]$ & 150 & 200 & 250 & 300 & 350 \\
\hline$\sigma_{Z H_{2}} / s_{\alpha}^{2}[\mathrm{fb}]$ & 52.9 & 43.0 & 32.1 & 21.4 & 11.9 \\
\hline$\epsilon_{S}[\%]$ & 87 & 86 & 85 & 79 & 12 \\
\hline \multicolumn{1}{|c|}{$Z \rightarrow \mu^{+} \mu^{-}$} & & & & & \\
$\epsilon_{B}[\%]$ & 0.046 & 0.094 & 0.16 & 0.31 & 0.11 \\
$s_{\alpha}^{\text {min. }}\left(\mathcal{L}=500 \mathrm{fb}^{-1}\right)$ & 0.11 & 0.15 & 0.20 & 0.30 & 0.78 \\
$s_{\alpha}^{\min .}\left(\mathcal{L}=1600 \mathrm{fb}^{-1}\right)$ & 0.084 & 0.11 & 0.15 & 0.22 & 0.58 \\
\hline \multicolumn{1}{|c}{$Z \rightarrow j j$} & & & & & \\
$\epsilon_{B}[\%]$ & 0.30 & 0.56 & 1.17 & 2.13 & 0.75 \\
$s_{\alpha}^{\text {min. }}\left(\mathcal{L}=500 \mathrm{fb}^{-1}\right)$ & 0.053 & 0.069 & 0.097 & 0.14 & 0.37 \\
$s_{\alpha}^{\min .}\left(\mathcal{L}=1600 \mathrm{fb}^{-1}\right)$ & 0.040 & 0.052 & 0.73 & 0.11 & 0.28 \\
\hline
\end{tabular}

Table 4. Signal and background efficiencies for detecting $e^{+} e^{-} \rightarrow Z H_{2}$ process at the ILC. Prospects for parameter constraints are also shown assuming $\mathcal{L}=500 \mathrm{fb}^{-1}$ and $1600 \mathrm{fb}^{-1}$.

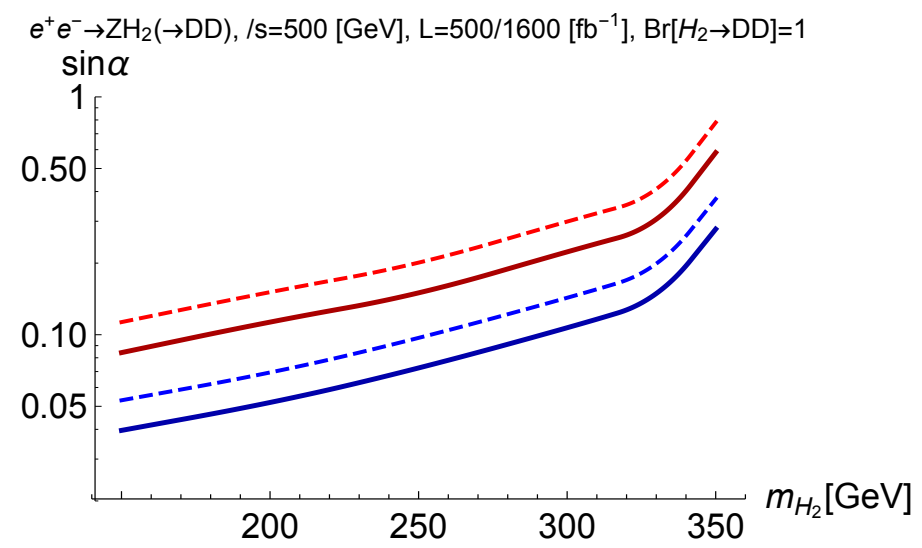

Figure 7. Contour plots for the discovery potential at $95 \%$ C.L. in $e^{+} e^{-} \rightarrow Z H_{2}$ searches using the invisible decay mode of $H_{2} \rightarrow D D$ at the ILC with $\sqrt{s}=500 \mathrm{GeV}$ and $\mathcal{L}=500$ (dashed), 1600 (solid) $\left[\mathrm{fb}^{-1}\right]$. Red contours are the limits by using the dimuon channel of $Z$-boson decay, and blue contours are the limits by using the dijet channel. $\operatorname{Br}\left(H_{2} \rightarrow D D\right)=1$ is assumed.

\subsection{Comparison with Higgs portal DM models within EFT}

As we have seen above, the collider signals of the Higgs portal DM models, which are UV-completed to preserve the gauge invariance, renormalizability and unitarity, are more complicated than the simple EFT-based models. The presence of the second Higgs boson which is inevitable to make the models with fermion or vector DM suitable with our requirement gives characteristic signals for the DM production as well as the new scalar itself.

For $m_{H_{2}} \gg \sqrt{s}$, the distribution cannot be distinguished from that of the EFT calculation. Thus, a long tail or a roll in the high- $t$ region does not immediately imply the unitary violation, but can be regarded as a characteristic signal of the fermion and vector 
DM models. However, the high-energy behavior of the model is completely different depending on whether the model is renormalizable or not as well as unitary or not. Collider phenomenology also depends on the details of the models, such as presences of new scalars, partners of DM, etc., and also constraints from the DM relic density, direct detections, etc. We emphasize that the interplay between these observations has to be performed in the model with renomalizability and unitarity to combine the model analyses in different scales.

Before closing this subsection, let us ask when we can ignore the 2nd scalar propagator in eq. (2.29), and use the EFT approach in which eq. (2.30) can be applied. Discussion at the ILC is simpler than at the LHC, since the CM energy $\sqrt{s}$ is fixed at the ILC. For a fixed $\sqrt{s}$, we can ignore the 2 nd scalar propagator in eq. (2.29) if $m_{H_{2}}^{2} \gg \sqrt{s}$. Then the effective Higgs portal Lagrangians, eqs. (1.2) and (1.3), might give reasonably good descriptions. However it is not true, since the invisible decay width of the $125 \mathrm{GeV}$ Higgs boson in case of Higgs portal VDM diverges when the VDM mass approaches zero, which is unphysical [31]: ${ }^{5}$

$$
\left(\Gamma_{h}^{\mathrm{inv}}\right)_{\mathrm{EFT}}=\frac{\lambda_{V H}^{2}}{128 \pi} \frac{v_{H}^{2} m_{h}^{3}}{m_{V}^{4}}\left(1-\frac{4 m_{V}^{2}}{m_{h}^{2}}+12 \frac{m_{V}^{4}}{m_{h}^{4}}\right)\left(1-\frac{4 m_{V}^{2}}{m_{h}^{2}}\right)^{1 / 2}
$$

On the other hand, it is perfectly finite in the full renormalizable and unitary model, since $m_{V}=g_{V} v_{\varphi} / 2[31]$ :

$$
\Gamma_{h}^{\text {inv }}=\frac{g_{V}^{2}}{32 \pi} \frac{m_{h}^{3}}{m_{V}^{2}}\left(1-\frac{4 m_{V}^{2}}{m_{h}^{2}}+12 \frac{m_{V}^{4}}{m_{h}^{4}}\right)\left(1-\frac{4 m_{V}^{2}}{m_{h}^{2}}\right)^{1 / 2} \sin ^{2} \alpha
$$

Note that there is more parameter, $\alpha$, in eq. (4.8), compared with eq. (4.7) for the invisible decay width in the VDM EFT with Higgs portal. For massive VDM, $v_{\varphi} \neq 0$ so that eq. (4.8) never diverges when $m_{V}$ becomes very light in the limit $g_{V} \rightarrow 0$. From the usual EFT view point, eq. (1.3) should be good at low energy as long as $m_{H_{2}} \gg m_{H_{1}}$, which however is not the case for the Higgs invisible decay width. It is not clear a priori when and where the EFT descriptions would fail in this particular physical quantity. Based on this example, it would be safer to work in the minimal renormalizable and unitary models for fermion and vector DM with Higgs portal.

\section{Conclusion}

In this paper, we have performed the detailed study of Higgs portal scalar, fermion and vector DM models at the ILC. We consider the renormalizable, unitary and gauge invariant models, and compare the results with those obtained within the effective field theories for the Higgs portal fermion and vector DM models. For the singlet fermion and vector DM cases, the force mediator involves two scalar propagators, the SM-like Higgs boson and the dark Higgs boson. We have shown that their interference generates interesting and important patterns in the mono- $Z$ plus missing $E_{T}$ signatures at the ILC, and the results are completely different from those obtained within the EFT. Compared with the EFT,

\footnotetext{
${ }^{5}$ The invisible decay widths in ref. [31] should be multiplied by $\sin ^{2} \alpha$ and $\cos ^{2} \alpha$ in order that we get the physical invisible widths of $H_{1}$ and $H_{2}$, respectively.
} 
our models have at least two extra parameters, the singlet-like scalar mass $m_{H_{2}}$ and the scalar mixing angle $\alpha$. These parameters are largely unknown yet, except that $\sin \alpha \lesssim 0.53$ from the current LHC data. The mixing angle $\alpha$ can be probed at an accuracy of $\mathcal{O}(1 \%)$ or better by precision measurement of the Higgs boson couplings at the ILC. By observing the DM pair production and the effects of the second Higgs boson to it, model parameters can be further constrained.

In addition, as we have shown in section 3 , it would be possible to distinguish the spin of DM in the Higgs portal scenarios, if the shape of the recoil-mass distribution could be observed. To achieve this, one needs large number of events and careful treatment of signal and background analysis, thus it is more difficult than finding the evidence and measuring the masses. However, we emphasize this possibility as an theoretical concept. Otherwise it would be extremely difficult to distinguish them. Detailed simulation analysis for the significance of separating different spin ansatz is beyond the scope of this paper. An analysis at the LHC in the same philosophy will be addressed in a separate publication [35].

\section{Acknowledgments}

We are grateful to Seungwon Baek, Bhaskar Dutta, Tathagata Ghosh and Teruki Kamon, Alexander Natale, Myeonghun Park, Wan-Il Park and Chaehyun Yu for discussions on the topics discussed in this paper. This work is supported in part by National Research Foundation of Korea (NRF) Research Grant NRF-2015R1A2A1A05001869, and by the NRF grant funded by the Korea government (MSIP) (No. 2009-0083526) through Korea Neutrino Research Center at Seoul National University (P.K.).

Open Access. This article is distributed under the terms of the Creative Commons Attribution License (CC-BY 4.0), which permits any use, distribution and reproduction in any medium, provided the original author(s) and source are credited.

\section{References}

[1] S. Kanemura, S. Matsumoto, T. Nabeshima and N. Okada, Can WIMP dark matter overcome the nightmare scenario?, Phys. Rev. D 82 (2010) 055026 [arXiv:1005.5651] [INSPIRE].

[2] O. Lebedev, H.M. Lee and Y. Mambrini, Vector Higgs-portal dark matter and the invisible Higgs, Phys. Lett. B 707 (2012) 570 [arXiv:1111.4482] [INSPIRE].

[3] A. Djouadi, O. Lebedev, Y. Mambrini and J. Quevillon, Implications of LHC searches for Higgs-portal dark matter, Phys. Lett. B 709 (2012) 65 [arXiv:1112.3299] [INSPIRE].

[4] L. Lopez-Honorez, T. Schwetz and J. Zupan, Higgs portal, fermionic dark matter and a Standard Model like Higgs at 125 GeV, Phys. Lett. B 716 (2012) 179 [arXiv:1203.2064] [INSPIRE].

[5] M. Endo and Y. Takaesu, Heavy WIMP through Higgs portal at the LHC, Phys. Lett. B 743 (2015) 228 [arXiv:1407.6882] [INSPIRE].

[6] N. Craig, H.K. Lou, M. McCullough and A. Thalapillil, The Higgs portal above threshold, JHEP 02 (2016) 127 [arXiv: 1412.0258] [INSPIRE]. 
[7] A. Beniwal et al., Combined analysis of effective Higgs portal dark matter models, Phys. Rev. D 93 (2016) 115016 [arXiv: 1512.06458] [INSPIRE].

[8] H. Han, J.M. Yang, Y. Zhang and S. Zheng, Collider signatures of Higgs-portal scalar dark matter, Phys. Lett. B 756 (2016) 109 [arXiv:1601.06232] [INSPIRE].

[9] ATLAS collaboration, Observation of a new particle in the search for the Standard Model Higgs boson with the ATLAS detector at the LHC, Phys. Lett. B 716 (2012) 1 [arXiv: 1207.7214] [INSPIRE].

[10] CMS collaboration, Observation of a new boson at a mass of $125 \mathrm{GeV}$ with the CMS experiment at the LHC, Phys. Lett. B 716 (2012) 30 [arXiv:1207.7235] [INSPIRE].

[11] CMS collaboration, Observation of a new boson with mass near $125 \mathrm{GeV}$ in pp collisions at $\sqrt{s}=7$ and $8 \mathrm{TeV}$, JHEP 06 (2013) 081 [arXiv:1303.4571] [INSPIRE].

[12] H. Baer et al., The International Linear Collider technical design report - volume 2: physics, arXiv:1306.6352 [INSPIRE].

[13] K. Fujii et al., Physics case for the International Linear Collider, arXiv:1506.05992 [INSPIRE].

[14] T. Barklow et al., ILC operating scenarios, arXiv:1506.07830 [INSPIRE].

[15] S. Baek, P. Ko and W.-I. Park, Search for the Higgs portal to a singlet fermionic dark matter at the LHC, JHEP 02 (2012) 047 [arXiv:1112.1847] [INSPIRE].

[16] S. Baek, P. Ko, W.-I. Park and E. Senaha, Vacuum structure and stability of a singlet fermion dark matter model with a singlet scalar messenger, JHEP 11 (2012) 116 [arXiv: 1209.4163] [INSPIRE].

[17] Y. Farzan and A.R. Akbarieh, VDM: a model for vector dark matter, JCAP 10 (2012) 026 [arXiv: 1207.4272] [INSPIRE].

[18] S. Baek, P. Ko, W.-I. Park and E. Senaha, Higgs portal vector dark matter: revisited, JHEP 05 (2013) 036 [arXiv: 1212.2131] [INSPIRE].

[19] V. Silveira and A. Zee, Scalar phantoms, Phys. Lett. B 161 (1985) 136 [INSPIRE].

[20] C.P. Burgess, M. Pospelov and T. ter Veldhuis, The minimal model of nonbaryonic dark matter: a singlet scalar, Nucl. Phys. B 619 (2001) 709 [hep-ph/0011335] [INSPIRE].

[21] A. Djouadi, A. Falkowski, Y. Mambrini and J. Quevillon, Direct detection of Higgs-portal dark matter at the LHC, Eur. Phys. J. C 73 (2013) 2455 [arXiv:1205.3169] [INSPIRE].

[22] S. Baek, P. Ko and W.-I. Park, Local $Z_{2}$ scalar dark matter model confronting galactic GeV-scale $\gamma$-ray, Phys. Lett. B 747 (2015) 255 [arXiv:1407.6588] [InSPIRE].

[23] P. Ko and Y. Tang, Self-interacting scalar dark matter with local $Z_{3}$ symmetry, JCAP 05 (2014) 047 [arXiv: 1402.6449] [InSPIRE].

[24] M. Duch, B. Grzadkowski and M. McGarrie, A stable Higgs portal with vector dark matter, JHEP 09 (2015) 162 [arXiv:1506.08805] [INSPIRE].

[25] A. DiFranzo, P.J. Fox and T.M.P. Tait, Vector dark matter through a radiative Higgs portal, JHEP 04 (2016) 135 [arXiv: 1512.06853] [INSPIRE].

[26] S. Baek, P. Ko and W.-I. Park, Hidden sector monopole, vector dark matter and dark radiation with Higgs portal, JCAP 10 (2014) 067 [arXiv:1311.1035] [INSPIRE]. 
[27] P. Ko, W.-I. Park and Y. Tang, Higgs portal vector dark matter for GeV scale $\gamma$-ray excess from galactic center, JCAP 09 (2014) 013 [arXiv: 1404.5257] [INSPIRE].

[28] P. Ko and Y. Tang, Galactic center $\gamma$-ray excess in hidden sector DM models with dark gauge symmetries: local $Z_{3}$ symmetry as an example, JCAP 01 (2015) 023 [arXiv:1407.5492] [INSPIRE].

[29] P. Ko and Y. Tang, Dark Higgs channel for FERMI GeV $\gamma$-ray excess, JCAP 02 (2016) 011 [arXiv: 1504.03908] [INSPIRE].

[30] P. Ko and W.-I. Park, Higgs-portal assisted Higgs inflation with a large tensor-to-scalar ratio, arXiv:1405.1635 [INSPIRE].

[31] S. Baek, P. Ko and W.-I. Park, Invisible Higgs decay width vs. dark matter direct detection cross section in Higgs portal dark matter models, Phys. Rev. D 90 (2014) 055014 [arXiv: 1405.3530] [INSPIRE].

[32] S. Choi, S. Jung and P. Ko, Implications of LHC data on $125 \mathrm{GeV}$ Higgs-like boson for the Standard Model and its various extensions, JHEP 10 (2013) 225 [arXiv:1307.3948] [INSPIRE].

[33] K. Cheung, P. Ko, J.S. Lee and P.-Y. Tseng, Bounds on Higgs-portal models from the LHC Higgs data, JHEP 10 (2015) 057 [arXiv:1507.06158] [INSPIRE].

[34] K. Cheung, P. Ko, J.S. Lee, J. Park and P.-Y. Tseng, A Higgcision study on the $750 \mathrm{GeV}$ di-photon resonance and $125 \mathrm{GeV}$ SM Higgs boson with the Higgs-singlet mixing, arXiv: 1512.07853 [INSPIRE].

[35] B. Dutta, T. Ghosh, T. Kamon, P. Ko and H. Yokoya, work in preparation.

[36] S. Baek, P. Ko, M. Park, W.-I. Park and C. Yu, Beyond the dark matter effective field theory and a simplified model approach at colliders, Phys. Lett. B 756 (2016) 289 [arXiv: 1506.06556] [INSPIRE].

[37] S. Matsumoto et al., Observing the coupling between dark matter and Higgs boson at the ILC, arXiv: 1006.5268 [INSPIRE].

[38] S. Kanemura, S. Matsumoto, T. Nabeshima and H. Taniguchi, Testing Higgs portal dark matter via Z fusion at a linear collider, Phys. Lett. B 701 (2011) 591 [arXiv:1102.5147] [INSPIRE].

[39] Z. Chacko, Y. Cui and S. Hong, Exploring a dark sector through the Higgs portal at a lepton collider, Phys. Lett. B 732 (2014) 75 [arXiv:1311.3306] [INSPIRE].

[40] M.A. Fedderke, T. Lin and L.-T. Wang, Probing the fermionic Higgs portal at lepton colliders, JHEP 04 (2016) 160 [arXiv: 1506. 05465] [INSPIRE].

[41] J.R. Andersen, M. Rauch and M. Spannowsky, Dark sector spectroscopy at the ILC, Eur. Phys. J. C 74 (2014) 2908 [arXiv: 1308.4588] [inSPIRE].

[42] CMS collaboration, Precise determination of the mass of the Higgs boson and tests of compatibility of its couplings with the Standard Model predictions using proton collisions at 7 and 8 TeV, Eur. Phys. J. C 75 (2015) 212 [arXiv:1412.8662] [InSPIRE].

[43] ATLAS collaboration, Measurements of the Higgs boson production and decay rates and coupling strengths using pp collision data at $\sqrt{s}=7$ and $8 \mathrm{TeV}$ in the ATLAS experiment, Eur. Phys. J. C 76 (2016) 6 [arXiv:1507.04548] [InSPIRE]. 
[44] ATLAS and CMS collaborations, Measurements of the Higgs boson production and decay rates and constraints on its couplings from a combined ATLAS and CMS analysis of the LHC pp collision data at $\sqrt{s}=7$ and 8 TeV, ATLAS-CONF-2015-044, CERN, Geneva Switzerland (2015).

[45] T. Hambye, Hidden vector dark matter, JHEP 01 (2009) 028 [arXiv:0811.0172] [INSPIRE].

[46] M. Asano et al., Discrimination of new physics models with the International Linear Collider, Phys. Rev. D 84 (2011) 115003 [arXiv:1106.1932] [INSPIRE].

[47] ATLAS collaboration, Search for invisible decays of a Higgs boson using vector-boson fusion in pp collisions at $\sqrt{s}=8 \mathrm{TeV}$ with the ATLAS detector, JHEP 01 (2016) 172 [arXiv: 1508.07869] [INSPIRE].

[48] ATLAS collaboration, Constraints on new phenomena via Higgs boson couplings and invisible decays with the ATLAS detector, JHEP 11 (2015) 206 [arXiv:1509.00672] [INSPIRE].

[49] CMS collaboration, Search for invisible decays of Higgs bosons in the vector boson fusion production mode, CMS-PAS-HIG-14-038, CERN, Geneva Switzerland (2014).

[50] CMS collaboration, A combination of searches for the invisible decays of the Higgs boson using the CMS detector, CMS-PAS-HIG-15-012, CERN, Geneva Switzerland (2015).

[51] Particle Data Group collaboration, K.A. Olive et al., Review of particle physics, Chin. Phys. C 38 (2014) 090001 [INSPIRE].

[52] J. Alwall et al., The automated computation of tree-level and next-to-leading order differential cross sections and their matching to parton shower simulations, JHEP 07 (2014) 079 [arXiv: 1405.0301] [INSPIRE]. 